TRANSACTIONS OF THE

AMERICAN MATHEMATICAL SOCIETY

Volume 356, Number 1, Pages 371-392

S 0002-9947(03)03391-9

Article electronically published on August 25, 2003

\title{
SLOPES OF VECTOR BUNDLES ON PROJECTIVE CURVES AND APPLICATIONS TO TIGHT CLOSURE PROBLEMS
}

\author{
HOLGER BRENNER
}

\begin{abstract}
We study different notions of slope of a vector bundle over a smooth projective curve with respect to ampleness and affineness in order to apply this to tight closure problems. This method gives new degree estimates from above and from below for the tight closure of a homogeneous $R_{+}$-primary ideal in a two-dimensional normal standard-graded algebra $R$ in terms of the minimal and the maximal slope of the sheaf of relations for some ideal generators. If moreover this sheaf of relations is semistable, then both degree estimates coincide and we get a vanishing type theorem.
\end{abstract}

\section{INTRODUCTION}

In this paper we continue the study of tight closure problems started in 3 of a two-dimensional normal standard-graded $K$-algebra $R$ in terms of the corresponding projective bundles and subbundles on the corresponding projective curve $Y=\operatorname{Proj} R$. A system of $R_{+}$-primary homogeneous elements $f_{1}, \ldots, f_{n} \in R$ leads to the locally free sheaf of relations $\mathcal{R}$ on $Y$, and a further homogeneous element yields an extension $\mathcal{R} \rightarrow \mathcal{R}^{\prime}$, which itself gives the projective subbundle $\mathbb{P}(\mathcal{F}) \subset \mathbb{P}\left(\mathcal{F}^{\prime}\right)$, where $\mathcal{F}=\mathcal{R}^{\vee}$ and $\mathcal{F}^{\prime}=\mathcal{R}^{\prime \vee}$.

The fundamental observation of [3] is that $f_{0}$ belongs to the tight closure of the ideal $\left(f_{1}, \ldots, f_{n}\right)$, that is, $f_{0} \in\left(f_{1}, \ldots, f_{n}\right)^{*}$ holds if and only if the open subset $\mathbb{P}\left(\mathcal{F}^{\prime}\right)-\mathbb{P}(\mathcal{F})$ is not an affine scheme. This link rests upon the reinterpretation of tight closure as solid closure in positive characteristic; see 14 for this notion and [15] and [16] for background on the theory of tight closure (in characteristic zero we work throughout this paper with the notion of solid closure). This gives a powerful geometric tool to study tight closure problems; see also 3 for the general setting and 4] for the proof that tight closure and plus closure are the same for $R_{+}$-primary ideals in a normal coordinate ring of an elliptic curve over a field of positive characteristic.

The theme of this paper is the minimal and the maximal slope of a locally free sheaf $\mathcal{G}$ on a smooth projective curve $Y$ and how these invariants and the corresponding properties such as semistability and ampleness are related to the properties which are of interest from the tight closure point of view. We recall and extend the necessary definitions and facts in section 1. In section 2 we consider criteria for ample bundles. Our main criterion, which is essentially due to Barton ([2, Theorem 2.1]), is that $\mathcal{G}$ is ample if and only if $\bar{\mu}_{\min }(\mathcal{G})>0$ (Theorem 2.3 ),

Received by the editors May 21, 2002 and, in revised form, February 19, 2003.

2000 Mathematics Subject Classification. Primary 13A35, $14 \mathrm{H} 60$.

(C)2003 American Mathematical Society 
where $\bar{\mu}_{\min }(\mathcal{G})$ is a variant of the minimal slope which also takes into account the behavior under finite mappings $Z \rightarrow Y$.

In section 3 we study sufficient conditions for the complement $\mathbb{P}\left(\mathcal{G}^{\prime}\right)-\mathbb{P}(\mathcal{G})$ to be affine, where $0 \rightarrow \mathcal{O}_{Y} \rightarrow \mathcal{G}^{\prime} \rightarrow \mathcal{G} \rightarrow 0$ is an extension given by a cohomology class $c \in H^{1}\left(Y, \mathcal{G}^{\vee}\right)$. From the ampleness criterion it easily follows in characteristic 0 that for $\mu(\mathcal{G})>0$ and $c \neq 0$ the complement $\mathbb{P}\left(\mathcal{G}^{\prime}\right)-\mathbb{P}(\mathcal{G})$ is affine (Corollary 3.2). This is also true if there exists a sheaf homomorphism $\varphi: \mathcal{G}^{\vee} \rightarrow \mathcal{T}$ such that $0 \neq \varphi(c) \in H^{1}(Y, \mathcal{T})$ and $\mathcal{T}$ is semistable of negative slope (Theorem 3.4). Our main result of section 4 is that $\mathbb{P}\left(\mathcal{G}^{\prime}\right)-\mathbb{P}(\mathcal{G})$ is not affine under the condition that $\bar{\mu}_{\max }(\mathcal{G}) \leq 0$ (Theorem 4.4). Sections 1 - 4 may be read without any knowledge of tight closure theory.

In section 5 we recall briefly how vector bundles arise from tight closure problems referring to [3] for details and proofs. In sections 6 - 9] we apply our results to tight closure problems. The slope invariants $\bar{\mu}_{\max }$ and $\bar{\mu}_{\min }$ of the relation bundle corresponding to ideal generators $f_{1}, \ldots, f_{n}$ of an $R_{+}$-primary ideal give important new degree estimates for $\left(f_{1}, \ldots, f_{n}\right)^{*}$.

We show that the condition $\operatorname{deg}\left(f_{0}\right) \geq \bar{\mu}_{\max }\left(f_{1}, \ldots, f_{n}\right) / \operatorname{deg}\left(\mathcal{O}_{Y}(1)\right)$ forces $f_{0} \in$ $\left(f_{1}, \ldots, f_{n}\right)^{*}$ (Theorem6.4 $)$. From this we derive that $R_{m} \subseteq\left(f_{1}, \ldots, f_{n}\right)^{*}$, whenever $m$ is greater than or equal to the sum of the two biggest degrees of the $f_{i}$ (Corollary 6.7), which improves slightly the bound $2 \max d_{i}$ of Smith given in [26, Proposition $3.1]$.

In the other direction we prove (if the characteristic of the field is 0 or $p \gg 0$ ) that if $\operatorname{deg}\left(f_{0}\right)<\bar{\mu}_{\min }\left(f_{1}, \ldots, f_{n}\right) / \operatorname{deg}\left(\mathcal{O}_{Y}(1)\right)$, then $f_{0} \in\left(f_{1}, \ldots, f_{n}\right)^{*}$ is only possible if $f_{0} \in\left(f_{1}, \ldots, f_{n}\right)$ already holds (Theorem 7.3 ). If the sheaf of relations splits into invertible sheaves, then the two bounds are easy to compute as the minimum (maximum) of the degrees of the summands. Moreover, in this splitting situation we can give a numerical criterion for tight closure (Theorem [7.5).

In section 8 we study the situation where the minimal and the maximal slope coincide. In this case the sheaf of relations is semistable, and we get the equality $\left(f_{1}, \ldots, f_{n}\right)^{*}=\left(f_{1}, \ldots, f_{n}\right)+R_{\geq k}$, where $k=\left\lceil\frac{d_{1}+\ldots+d_{n}}{n-1}\right\rceil$ (Theorems 8.1 and 8.4). This is a (so-called) vanishing type theorem in the sense of [17 and generalizes the vanishing theorem in the parameter case $(n=2)$, where the sheaf of relations is invertible, hence of course semistable.

In section 9 we concentrate on the case $n=3$. We give bounds for $\mu_{\max }$ and $\mu_{\text {min }}$ deriving from known results about the $e$-invariant of a ruled surface (Theorem 9.1). Under the condition that the sheaf of relations is indecomposable we obtain degree bounds for inclusion and exclusion which are quite near to $k=\left(d_{1}+d_{2}+d_{3}\right) / 2$, the difference is at most $(g-1) / \operatorname{deg}\left(\mathcal{O}_{Y}(1)\right)$, where $g$ is the genus and of the curve (Corollary 9.2).

\section{Slope OF BUNDLES}

In this section we recall the notion of the slope of bundles and various related concepts such as semistable sheaves and the Harder-Narasimhan filtration, which we will need in the sequel. Our main references are [9], [18], [21, Ch. 6.4], 22] and 25].

Let $\mathcal{E}$ denote a locally free sheaf on a smooth projective curve $Y$ over an algebraically closed field $K$. The degree of $\mathcal{E}$ is defined by $\operatorname{deg}(\mathcal{E})=\operatorname{deg}\left(\bigwedge^{r}(\mathcal{E})\right)$, where $r$ is the rank of $\mathcal{E}$. If $\mathbb{P}(\mathcal{E})=\operatorname{Proj} \oplus_{k} S^{k}(\mathcal{E})$ is the corresponding projective bundle 
of dimension $r$ and if $\xi$ denotes the divisor class corresponding to the relatively very ample invertible sheaf $\mathcal{O}_{\mathbb{P}(\mathcal{E})}(1)$, then $\operatorname{deg}(\mathcal{E})=\xi^{r}$ also equals the top self intersection number; see [21, Lemma 6.4.10]. The number $\operatorname{deg}(\mathcal{E}) / r$ ! is also the coefficient of $k^{r}$ in the Euler-Hilbert polynomial $\chi\left(\mathcal{O}_{\mathbb{P}(\mathcal{E})}(k)\right)$, which equals $\chi\left(S^{k}(\mathcal{E})\right)$.

The slope of a locally free sheaf $\mathcal{E}$ is defined by $\mu(\mathcal{E})=\operatorname{deg}(\mathcal{E}) / \operatorname{rk}(\mathcal{E})$. A locally free sheaf $\mathcal{E}$ is called semistable, if for every locally free quotient sheaf $\mathcal{E} \rightarrow \mathcal{Q} \rightarrow 0$ the inequality $\mu(\mathcal{Q}) \geq \mu(\mathcal{E})$ holds. This is equivalent to the property that for every locally free subsheaf $\mathcal{T} \subseteq \mathcal{E}$ the inequality $\mu(\mathcal{T}) \leq \mu(\mathcal{E})$ holds.

Every locally free sheaf $\mathcal{E}$ has a unique Harder-Narasimhan Filtration. This is a filtration of locally free subsheaves

$$
0=\mathcal{E}_{0} \subset \mathcal{E}_{1} \subset \ldots \subset \mathcal{E}_{s}=\mathcal{E}
$$

such that $\mathcal{E}_{i} / \mathcal{E}_{i-1}$ is semistable for every $i=1, \ldots, s$. $\mathcal{E}_{1}$ is called the maximal destabilizing subsheaf. The slopes of these semistable quotients form a decreasing chain $\mu_{1}>\ldots>\mu_{s} . \quad \mu_{\min }(\mathcal{E})=\mu_{s}=\mu\left(\mathcal{E} / \mathcal{E}_{s-1}\right)$ is called the minimal slope and $\mu_{\max }(\mathcal{E})=\mu_{1}(\mathcal{E})$ is called the maximal slope. This is the same as $\mu_{\min }(\mathcal{E})=$ $\min \{\mu(\mathcal{Q}): \mathcal{E} \rightarrow \mathcal{Q} \rightarrow 0\}$. For the dual sheaf we have $\mu_{\max }\left(\mathcal{E}^{\vee}\right)=-\mu_{\min }(\mathcal{E})$. If $\mu_{\min }(\mathcal{E})>\mu_{\max }(\mathcal{F})$, then $\operatorname{Hom}(\mathcal{E}, \mathcal{F})=0$. In particular, if $\mu_{\max }(\mathcal{F})<0$, then $\Gamma(Y, \mathcal{F})=0$.

If $\varphi: Z \rightarrow Y$ is a finite $K$-morphism between smooth projective curves over the algebraically closed field $K$, then $\mu\left(\varphi^{*}(\mathcal{E})\right)=\operatorname{deg}(\varphi) \mu(\mathcal{E})$. If $\varphi$ is separable, then the pull-back $\varphi^{*}(\mathcal{E})$ of a semistable sheaf $\mathcal{E}$ on $Y$ is again semistable; see 22 Proposition 3.2]. Hence in the separable case the Harder-Narasimhan filtration of $\varphi^{*}(\mathcal{E})$ is just the pull-back of the Harder-Narasimhan filtration of $\mathcal{E}$, and also $\mu_{\max }$ and $\mu_{\min }$ transform in the same way as $\mu$ does.

In the non-separable case this is not true at all, and the notion of semistability needs to be refined. A locally free sheaf $\mathcal{E}$ on $Y$ is called strongly semistable, if for every finite $K$-morphism $\varphi: Z \rightarrow Y$ the pull-back $\varphi^{*}(\mathcal{E})$ is again semistable. In characteristic zero, this is the same as being semistable, and in positive characteristic it is given by the property that the pull-back under every $K$-linear Frobenius morphism is semistable; see [22, Proposition 5.1]. This difficulty in positive characteristic is one motivation for the following definition.

Definition 1.1. Let $Y$ denote a smooth projective curve over an algebraically closed field and let $\mathcal{E}$ denote a locally free sheaf. Then we define

$$
\bar{\mu}_{\max }(\mathcal{E})=\sup \left\{\frac{\mu_{\max }\left(\varphi^{*} \mathcal{E}\right)}{\operatorname{deg}(\varphi)} \mid \varphi: Z \rightarrow Y \text { finite dominant } K \text {-morphism }\right\}
$$

and

$$
\bar{\mu}_{\min }(\mathcal{E})=\inf \left\{\frac{\mu_{\min }\left(\varphi^{*} \mathcal{E}\right)}{\operatorname{deg}(\varphi)} \mid \varphi: Z \rightarrow Y \text { finite dominant } K \text {-morphism }\right\} .
$$

Remark 1.2. It is enough to consider in the previous definition only $K$-linear Frobenius morphisms, since every morphism factors through a separable map and a Frobenius and the maximal and minimal slope behave well with respect to separable morphisms.

We will see in Remark 2.4 that $\bar{\mu}_{\min }(\mathcal{E})$ is bounded from below, hence these numbers exist, but it is not clear whether they are obtained. An equivalent question is whether one may find a sufficiently high Frobenius power such that the HarderNarasimhan filtration of $\varphi^{*}(\mathcal{E})$ consists of strongly semistable quotients. 
We will also need the following definition; compare with [19, 20] and [23] for related invariants of $\mathcal{E}$ and $\mathbb{P}(\mathcal{E})$.

Definition 1.3. Let $\mathcal{E}$ denote a locally free sheaf on a smooth projective curve and let $1 \leq s \leq \operatorname{rk}(\mathcal{E})$. We set

$$
\rho_{s}(\mathcal{E})=\min \{\operatorname{deg}(\mathcal{Q}): \mathcal{E} \rightarrow \mathcal{Q} \rightarrow 0, \quad \mathcal{Q} \text { is locally free and } \operatorname{rk}(\mathcal{Q})=s\} .
$$

\section{Ampleness CRITERIA FOR VeCtOR BUndLES OVER PROJeCtive CURVES}

Recall that a locally free sheaf $\mathcal{G}$ on a scheme $Y$ is called ample if the invertible sheaf $\mathcal{O}_{\mathbb{P}(\mathcal{G})}(1)$ on the projective bundle $\mathbb{P}(\mathcal{G})=\operatorname{Proj} \oplus_{n} S^{n}(\mathcal{G})$ is ample. In characteristic zero we have the following linear criterion of Hartshorne-Miyaoka for ample sheaves over a curve.

Theorem 2.1. Let $Y$ denote a smooth projective curve over an algebraically closed field of characteristic 0 . Let $\mathcal{G}$ denote a locally free sheaf on $Y$ and $\mathbb{P}(\mathcal{G})$ the corresponding projective bundle, and let $\xi$ denote the divisor class corresponding to $\mathcal{O}_{\mathbb{P}(\mathcal{G})}(1)$. Then the following are equivalent:

(i) The sheaf $\mathcal{G}$ is ample.

(ii) For every projective subbundle $\mathbb{P}(\mathcal{Q}) \subseteq \mathbb{P}(\mathcal{G})$ of dimension s we have $\mathbb{P}(\mathcal{Q})$. $\xi^{s}$ $>0$.

(iii) For every locally free quotient sheaf $\mathcal{G} \rightarrow \mathcal{Q} \rightarrow 0, \mathcal{Q} \neq 0$, we have $\operatorname{deg}(\mathcal{Q})>$ 0 .

(iv) The minimal slope is $\mu_{\min }(\mathcal{G})>0$.

Proof. The equivalence of (ii), (iii) and (iv) is clear since $\mathbb{P}(\mathcal{Q}) \cdot \xi^{s}=\left(\left.\xi\right|_{\mathbb{P}(\mathcal{Q})}\right)^{s}=$ $\operatorname{deg}(\mathcal{Q})$. (i) $\Rightarrow$ (ii) follows from the Nakai-criterion; for the other direction see 11 Theorem 2.4], 22, Corollary 3.5] or [21, Theorem 6.4.15].

The minimal degree $\rho_{1}(\mathcal{G})$ of a quotient invertible sheaf of $\mathcal{G}$ must fulfill a stronger condition to guarantee that $\mathcal{G}$ is ample.

Corollary 2.2. Let $Y$ denote a smooth projective curve of genus $g$ over an algebraically closed field of characteristic 0 . Let $\mathcal{G}$ denote a locally free sheaf of rank $r$ on $Y$ and suppose that $\rho_{1}(\mathcal{G})>\frac{r-1}{r} g$. Then $\mathcal{G}$ is ample.

Proof. This is deduced in [19, Proposition 2] from the theorem.

The conditions (ii)-(iv) in Theorem 2.1 are in positive characteristic not sufficient for ampleness. This is due to the fact that the pull-back of a semistable sheaf under a non-separable morphism need not be semistable anymore and the minimal slope may drop. It is also related to the failure of the vanishing theorem in tight closure theory in small positive characteristic. The following criterion is valid in every characteristic and is essentially due to Barton (see [2, Theorem 2.1]).

Theorem 2.3. Let $Y$ denote a smooth projective curve over an algebraically closed field $K$ of characteristic $p \geq 0$. Let $\mathcal{G}$ denote a locally free sheaf of rank $r$ on $Y$. Then the following are equivalent:

(i) The sheaf $\mathcal{G}$ is ample.

(ii) $\bar{\mu}_{\min }(\mathcal{G})>0$.

(iii) There exists $\epsilon>0$ such that for every finite $K$-morphism $\varphi: Y^{\prime} \rightarrow Y$ and every invertible quotient sheaf $\varphi^{*}(\mathcal{G}) \rightarrow \mathcal{M} \rightarrow 0$ the inequality $\frac{\operatorname{deg}(\mathcal{M})}{\operatorname{deg}(\varphi)} \geq$ $\epsilon>0$ holds. 
Proof. (i) $\Rightarrow$ (ii). Suppose that $\varphi: Y^{\prime} \rightarrow Y$ is finite, where $Y^{\prime}$ is another smooth projective curve, and let $\varphi^{*}(\mathcal{G}) \rightarrow \mathcal{H} \rightarrow 0$ be given, $s=\operatorname{rk}(\mathcal{H})$. We consider first the case $s=1$. Then $\mathcal{M}=\mathcal{H}$ is an invertible sheaf on $Y^{\prime}$ and the surjection $\varphi^{*}(\mathcal{G}) \rightarrow \mathcal{M} \rightarrow 0$ defines a section $s: Y^{\prime} \rightarrow \mathbb{P}\left(\varphi^{*}(\mathcal{G})\right)$ due to the correspondence described in [7, Proposition 4.2.3] and a curve $Z$ (its image) in $\mathbb{P}(\mathcal{G})$. The map $Y^{\prime} \rightarrow Z$ factors through the normalization of $Z$, hence $\mathcal{M}$ is defined already on this normalization. Therefore we may assume that $Y^{\prime}$ is the normalization of $Z$. The numerical class of the curve $Z$ in $\mathbb{P}(\mathcal{G})$ can be written as $a \xi^{r-1}+b \xi^{r-2} \cdot f$, where $\xi$ is again the divisor class corresponding to $\mathcal{O}_{\mathbb{P}(\mathcal{G})}(1), f$ is the class of a fiber $\mathbb{P}(\mathcal{G}) \rightarrow Y$ and $a, b \in \mathbb{Z}$. Furthermore $a$ equals the degree of $\varphi$. Therefore we have

$$
\operatorname{deg}(\mathcal{M})=Y^{\prime} \cdot s^{*}\left(\mathcal{O}_{\mathbb{P}(\mathcal{G})}(1)\right)=\left(a \xi^{r-1}+b \xi^{r-2} \cdot f\right) \cdot \xi=a \xi^{r}+b=a \operatorname{deg}(\mathcal{G})+b .
$$

Hence $\operatorname{deg}(\mathcal{M}) / \operatorname{deg}(\varphi)=\operatorname{deg}(\mathcal{G})+b / a$. Since $\mathcal{G}$ and hence $\xi$ is ample, there exists a number $\epsilon>0$ such that $\xi . Z \geq \epsilon\|Z\|$ holds for every curve, where $\|Z\|$ denotes any norm on Num $\otimes \mathbb{R}$; see [12, Theorem 8.1]. Hence

$$
\operatorname{deg}(\mathcal{G})+\frac{b}{a}=\frac{\xi \cdot Z}{a} \geq \frac{\epsilon \sqrt{a^{2}+b^{2}}}{a}=\epsilon \sqrt{1+\left(\frac{b}{a}\right)^{2}} \geq \epsilon .
$$

Now consider the general case. Since $\mathcal{G}$ is ample, also its wedge product $\bigwedge^{s} \mathcal{G}$ is ample, due to [10, Corollary 2.6]. The surjection $\varphi^{*}(\mathcal{G}) \rightarrow \mathcal{H} \rightarrow 0$ yields a surjection $\bigwedge^{s}\left(\varphi^{*} \mathcal{G}\right) \rightarrow \bigwedge^{s} \mathcal{H} \rightarrow 0 . \mathcal{M}=\bigwedge^{s} \mathcal{H}$ is invertible and since $\bigwedge^{s}\left(\varphi^{*} \mathcal{G}\right)$ is ample, there exists an $\epsilon_{s}$ such that $\operatorname{deg}\left(\bigwedge^{s} \mathcal{H}\right) / \operatorname{deg}(\varphi) \geq \epsilon_{s}>0$. Then $\bar{\mu}_{\min }(\mathcal{G}) \geq \min _{s} \epsilon_{s} / s>0$.

(ii) $\Rightarrow$ (iii) is a restriction to invertible quotient sheaves.

(iii) $\Rightarrow$ (i). Let $\xi$ denote the hypersection divisor corresponding to $\mathcal{O}_{\mathbb{P}(\mathcal{G})}(1)$. Due to the ampleness criterion of Seshadri (see [12, I $\S 7]$ ), it is enough to show that there exists an $\epsilon>0$ such that $\frac{\xi \cdot Z}{m(Z)} \geq \epsilon>0$ holds for every (effective) curve $Z$, where $m(Z)=\sup \left\{\operatorname{mult}_{P}(Z)\right\}$ is the maximal multiplicity of a point on $Z$. So suppose that $Z$ is an irreducible curve in $\mathbb{P}(\mathcal{G})$. If $Z$ lies in a fiber $F \cong \mathbb{P}^{r-1}$, then $\xi . Z=\operatorname{deg}(Z) \geq m(Z)$. Hence we may assume that $Z$ dominates the base. Let $Z^{\prime}$ be the normalization of $Z, i: Z^{\prime} \rightarrow \mathbb{P}(\mathcal{G})$ the corresponding mapping and let $\varphi: Z^{\prime} \rightarrow Y$ be the composition. Let $\varphi^{*} \mathcal{G} \rightarrow \mathcal{M} \rightarrow 0$ be the corresponding surjection onto the invertible sheaf $\mathcal{M}$.

The multiplicity $m(Z)$ is bounded above by $\operatorname{deg}(\varphi)$. Therefore we have

$$
\frac{\xi . Z}{m(Z)}=\frac{\operatorname{deg}(\mathcal{M})}{m(Z)} \geq \frac{\operatorname{deg}(\mathcal{M})}{\operatorname{deg}(\varphi)} \geq \epsilon>0 .
$$

Remark 2.4. If $\mathcal{G}$ is locally free on $Y$ and if $\mathcal{O}_{Y}(1)$ is an ample invertible sheaf on $Y$, then $\mathcal{G}(n)=\mathcal{G} \otimes \mathcal{O}_{Y}(n)$ corresponds to the invertible sheaf $\mathcal{O}_{\mathbb{P}(\mathcal{G})}(1) \otimes p^{*}\left(\mathcal{O}_{Y}(n)\right)$ on $\mathbb{P}(\mathcal{G})$; see [7, Proposition 4.1.4]. Choosing $n$ high enough, we may achieve that $\mathcal{G}(n)$ becomes ample. Since the slopes transform like $\mu_{\min }\left(\mathcal{G} \otimes \mathcal{L}^{k}\right)=\mu_{\min }(\mathcal{G})+k \operatorname{deg}(\mathcal{L})$, it follows that $\bar{\mu}_{\min }(\mathcal{G})=\bar{\mu}_{\min }\left(\mathcal{G} \otimes \mathcal{O}_{Y}(k)\right)-k \operatorname{deg}\left(\mathcal{O}_{Y}(1)\right)$ is bounded from below. Dually it follows that $\bar{\mu}_{\max }(\mathcal{S})$ is bounded from above, so both numbers exist (but it is not clear whether they are obtained).

Corollary 2.5. Let $Y$ denote a smooth projective curve over an algebraically closed field of characteristic $p \geq 0$. Let $\mathcal{G}$ denote a locally free sheaf on $Y$. Suppose that $\mathcal{G}$ is strongly semistable. Then $\mathcal{G}$ is ample if and only if its degree is positive. 
Proof. This follows directly from Theorem 2.3. For another proof see 22, Corollary 3.5 and $\S 5]$.

The ampleness of a locally free sheaf has also the following consequence on $\mu_{\max }$, which we will use in section 6

Corollary 2.6. Let $\mathcal{G}$ denote an ample locally free sheaf of rank $r$ on a smooth projective curve $Y$. Then we have the estimates $\left(\right.$ set $\left.\rho_{0}(\mathcal{G})=0\right)$

$$
\mu_{\max }(\mathcal{G}) \leq \max _{s=0, \ldots, r-1} \frac{\operatorname{deg}(\mathcal{G})-\rho_{s}(\mathcal{G})}{r-s} \leq \operatorname{deg}(\mathcal{G}) .
$$

Proof. Let $\mathcal{T} \subset \mathcal{G}$ denote a locally free subsheaf of positive rank with a short exact sequence $0 \rightarrow \mathcal{T} \rightarrow \mathcal{G} \rightarrow \mathcal{Q} \rightarrow 0, s=\operatorname{rk}(\mathcal{Q}), s=0, \ldots, r-1$. Then

$$
\mu(\mathcal{T})=\frac{\operatorname{deg}(\mathcal{T})}{r-s}=\frac{\operatorname{deg}(\mathcal{G})-\operatorname{deg}(\mathcal{Q})}{r-s} \leq \frac{\operatorname{deg}(\mathcal{G})-\rho_{s}(\mathcal{G})}{r-s}
$$

due to the definition of $\rho_{s}(\mathcal{E})$ as the minimum of $\operatorname{deg}(\mathcal{Q})$, where $\mathcal{Q}$ is a quotient sheaf of rank $s$. Since $\mathcal{G}$ is ample, it follows that $\rho_{s}(\mathcal{G})>0$, hence the estimate with $\operatorname{deg}(\mathcal{G})$ follows. (This last estimate holds also for $\bar{\mu}_{\max }$.)

If the bundle has rank two, the following theorem of Hartshorne-Mumford gives a satisfactory criterion for ampleness also in positive characteristic.

Theorem 2.7. Let $Y$ denote a smooth projective curve of genus $g$ defined over an algebraically closed field of characteristic $p>0$. Let $\mathcal{F}$ denote a locally free sheaf of rank two on $Y$. Suppose that $\operatorname{deg}(\mathcal{F})>\frac{2}{p}(g-1)$ and $\operatorname{deg}(\mathcal{L})>0$ for every invertible quotient sheaf $\mathcal{F} \rightarrow \mathcal{L} \rightarrow 0$. Then $\mathcal{F}$ is ample.

Proof. See [10, Proposition 7.5 and Corollary 7.7].

Remark 2.8. There exist more criteria for ample and very ample vector bundles on curves; see for example 1], [5], [19]. We omit them, since they don't seem to have implications on tight closure problems.

\section{CRiteria FOR AFFineness}

For ease of reference we fix the following situation.

Situation 3.1. Let $Y$ denote a smooth projective curve over an algebraically closed field $K$, let $\mathcal{S}$ denote a locally free sheaf on $Y$, and let $\mathcal{G}=\mathcal{S}^{\vee}$ be its dual sheaf. A cohomology class $c \in H^{1}(Y, \mathcal{S})=\operatorname{Ext}^{1}\left(\mathcal{O}_{Y}, \mathcal{S}\right)$ yields a short exact sequence

$$
0 \longrightarrow \mathcal{S} \longrightarrow \mathcal{S}^{\prime} \longrightarrow \mathcal{O}_{Y} \longrightarrow 0 \text {. }
$$

The dual sequence $0 \rightarrow \mathcal{O}_{Y} \rightarrow \mathcal{G}^{\prime} \rightarrow \mathcal{G} \rightarrow 0$ yields a projective subbundle $\mathbb{P}(\mathcal{G}) \subset$ $\mathbb{P}\left(\mathcal{G}^{\prime}\right)$ of codimension one.

Such a situation arises in particular from a homogeneous $R_{+}$-primary tight closure problem in a homogeneous coordinate ring $R$ of $Y$. This tight closure point of view leads to the question of whether the complement $\mathbb{P}\left(\mathcal{G}^{\prime}\right)-\mathbb{P}(\mathcal{G})$ is affine or not, in dependence of $\mathcal{S}$ and $c \in H^{1}(Y, \mathcal{S})$ (see section [5). Although in this section we consider mainly the case of characteristic 0 , subsequent results in positive characteristic are discussed in section 7 and section 8 . The ampleness criterion of Theorem 2.1 yields at once the following affineness criterion. 
Corollary 3.2. Let the notation be as in Situation 3.1 and suppose that $\operatorname{char}(K)=$ 0 . Suppose that $\mathcal{G}$ is ample - that is $\mu_{\min }(\mathcal{G})>0$ or equivalently $\mu_{\max }(\mathcal{S})<0$-and that $c \neq 0$. Then $\mathbb{P}\left(\mathcal{G}^{\prime}\right)-\mathbb{P}(\mathcal{G})$ is affine.

Proof. Since $\mathcal{G}$ is ample and since $0 \rightarrow \mathcal{O}_{Y} \rightarrow \mathcal{G}^{\prime} \rightarrow \mathcal{G} \rightarrow 0$ is a non-splitting short exact sequence, the sheaf $\mathcal{G}^{\prime}$ is also ample due to 6. Theorem 2.2] (here we use characteristic 0$)$. Hence $\mathbb{P}(\mathcal{G}) \subset \mathbb{P}\left(\mathcal{G}^{\prime}\right)$ is an ample divisor and its complement is affine.

Even if the divisor $\mathbb{P}(\mathcal{G}) \subset \mathbb{P}\left(\mathcal{G}^{\prime}\right)$ is not ample, the open subset $\mathbb{P}\left(\mathcal{G}^{\prime}\right)-\mathbb{P}(\mathcal{G})$ may be affine. We need the following lemma to obtain more general sufficient criteria for the affineness of the complement.

Lemma 3.3. Let $Y$ denote a scheme, let $\mathcal{S}$ and $\mathcal{T}$ be locally free sheaves on $Y$, and let $q: \mathcal{S} \rightarrow \mathcal{T}$ be a sheaf homomorphism. Let $c \in H^{1}(Y, \mathcal{S})$ with corresponding extension $0 \rightarrow \mathcal{S} \rightarrow \mathcal{S}^{\prime} \rightarrow \mathcal{O}_{Y} \rightarrow 0$ and let $q(c) \in H^{1}(Y, \mathcal{T})$ be its image with corresponding extension $0 \rightarrow \mathcal{T} \rightarrow \mathcal{T}^{\prime} \rightarrow \mathcal{O}_{Y} \rightarrow 0$.

If $\mathbb{P}\left(\left(\mathcal{T}^{\prime}\right)^{\vee}\right)-\mathbb{P}\left(\mathcal{T}^{\vee}\right)$ is affine, then also $\mathbb{P}\left(\left(\mathcal{S}^{\prime}\right)^{\vee}\right)-\mathbb{P}\left(\mathcal{S}^{\vee}\right)$ is affine.

Proof. See [4, Lemma 3.1].

Theorem 3.4. Let the notation be as in Situation 3.1 and suppose that $\operatorname{char}(K)=$ 0 . Suppose that there exists a semistable sheaf $\mathcal{T}$ of negative slope, $\mu(\mathcal{T})<0$, and a sheaf morphism $q: \mathcal{S} \rightarrow \mathcal{T}$ such that $q(c) \neq 0$ in $H^{1}(Y, \mathcal{T})$. Then the complement $\mathbb{P}\left(\mathcal{G}^{\prime}\right)-\mathbb{P}(\mathcal{G})$ is affine.

Proof. The sheaf $\mathcal{H}=\mathcal{T}^{\vee}$ is semistable of positive degree, hence ample due to Corollary 2.5 therefore $\mathbb{P}\left(\mathcal{H}^{\prime}\right)-\mathbb{P}(\mathcal{H})$ is affine due to Corollary 3.2. Therefore $\mathbb{P}\left(\mathcal{G}^{\prime}\right)-\mathbb{P}(\mathcal{G})$ is also affine due to Lemma 3.3 .

The first candidates of semistable sheaves to look at are $\mathcal{S} / \mathcal{S}_{s-1}$ (the semistable quotient of minimal slope) and invertible sheaves.

Corollary 3.5. Let the notation be as in Situation 3.1 and suppose that $\operatorname{char}(K)=$ 0 . Suppose that $\mu_{\min }(\mathcal{S})<0$ (or equivalently $\mu_{\max }(\mathcal{G})>0$ ) and that the image of $c \in H^{1}(Y, \mathcal{S})$ in the semistable quotient $\mathcal{Q}=\mathcal{S} / \mathcal{S}_{s-1}$ is $\neq 0$. Then $\mathbb{P}\left(\mathcal{G}^{\prime}\right)-\mathbb{P}(\mathcal{G})$ is affine.

Proof. Since $\mu(\mathcal{Q})=\mu_{\min }(\mathcal{S})<0$, the result follows from Theorem 3.4

Corollary 3.6. Let the notation be as in Situation 3.1 and suppose that $\operatorname{char}(K)=$ 0 . Suppose that $\mathcal{G}$ is not normalized, that is, there exists an invertible sheaf $\mathcal{L}$ of negative degree such that $H^{0}(Y, \mathcal{G} \otimes \mathcal{L}) \neq 0$. If $q: \mathcal{S} \rightarrow \mathcal{L}$ is a corresponding non-trivial map such that $0 \neq q(c) \in H^{1}(Y, \mathcal{L})$, then $\mathbb{P}\left(\mathcal{G}^{\prime}\right)-\mathbb{P}(\mathcal{G})$ is affine.

Proof. Note that $H^{0}(Y, \mathcal{G} \otimes \mathcal{L})=\operatorname{Hom}_{\mathcal{O}_{Y}}(\mathcal{S}, \mathcal{L})$, thus this follows again from Theorem 3.4.

Remark 3.7. In positive characteristic the assumption in Corollary 3.2 that $c \neq 0$ is too weak to ensure the ampleness of $\mathcal{G}^{\prime}$. We need the stronger condition that $0 \neq \varphi^{*}(c) \in H^{1}\left(Y^{\prime}, \varphi^{*}(\mathcal{S})\right)$ for every finite morphism $\varphi: Y^{\prime} \rightarrow Y$. In the situation coming from a forcing problem in tight closure, this condition means that $f_{0}$ does not belong to the plus closure.

However, if $\mathcal{G}$ is invertible and of positive degree, then the extension $\mathcal{G}^{\prime}$ corresponding to $0 \neq c \in H^{1}\left(Y, \mathcal{G}^{\vee}\right)$ is ample also in positive characteristic $p$ under the 
condition that $p \geq 2(g-1)$, where $g$ is the genus of the curve $Y$. This follows from the ampleness criterion of Hartshorne-Mumford for bundles of rank two; see Theorem 2.7.

From this it follows that Corollary 3.6 also holds in positive characteristic $p \gg 0$. For in this case the cohomology class $0 \neq q(c) \in H^{1}(Y, \mathcal{L})$ in the assumption of Corollary 3.6 gives rise to an ample sheaf $\left(\mathcal{L}^{\prime}\right)^{\vee}$, and then the open subset $\mathbb{P}\left(\left(\mathcal{L}^{\prime}\right)^{\vee}\right)-$ $\mathbb{P}\left(\mathcal{L}^{\vee}\right)$ is affine. The affineness of $\mathbb{P}\left(\mathcal{G}^{\prime}\right)-\mathbb{P}(\mathcal{G})$ then follows from Lemma 3.3.

If $\mathcal{G}$ is indecomposable of rank $r \geq 2$, then the degree of $\mathcal{G}$ must fulfill stronger conditions to ensure ampleness. The result [28, Theorem 25] suggests that the right inequality may be $\operatorname{deg}(\mathcal{G})>r(r-1)(g-1)+2 r(g-1) / p$ (or equivalently $\mu(\mathcal{G})>(g-1)(r+p / 2-1))$. Tango considers only the behavior of a cohomology class $c \in H^{1}(Y, \mathcal{S})$ under the Frobenius, but along these lines it should be possible to also deduce an ample criterion.

Example 3.8. Examples of semistable sheaves of positive degree which are not ample (or which are not strongly semistable) correspond to examples where the vanishing theorem for tight closure does not hold in small characteristic. Look at the examples in [11, §3] and [6, Lemma 2.4]) on the one hand and [17, Example 3.11] on the other hand.

\section{Criteria for $\mathbb{P}\left(\mathcal{G}^{\prime}\right)-\mathbb{P}(\mathcal{G})$ not to be affine}

We look again at Situation 3.1 , but now we look for criteria for $\mathbb{P}\left(\mathcal{G}^{\prime}\right)-\mathbb{P}(\mathcal{G})$ not to be affine. Since affine subsets contain no projective curves, we get the following easy criterion.

Proposition 4.1. Let the notation be as in Situation 3.1. Suppose that there exists a finite morphism $\varphi: Z \rightarrow Y$ such that $\varphi^{*}(c)=0$. Then $\mathbb{P}\left(\mathcal{G}^{\prime}\right)-\mathbb{P}(\mathcal{G})$ is not affine.

Proof. The condition means that the pull-back of $0 \rightarrow \mathcal{O}_{Y} \rightarrow \mathcal{G}^{\prime} \rightarrow \mathcal{G} \rightarrow 0$ splits on $Z$. The splitting surjection $\varphi^{*}\left(\mathcal{G}^{\prime}\right) \rightarrow \mathcal{O}_{Z} \rightarrow 0$ yields a section $Z \rightarrow \mathbb{P}\left(\varphi^{*}\left(\mathcal{G}^{\prime}\right)\right)$ disjoined to $\mathbb{P}\left(\varphi^{*}(\mathcal{G})\right)$ and hence there exists a projective curve inside $\mathbb{P}\left(\mathcal{G}^{\prime}\right)-\mathbb{P}(\mathcal{G})$.

Remark 4.2. If the complement of an effective Weil divisor $D$ on a normal projective variety $X$ of dimension $d$ is affine, then $\Gamma\left(X-D, \mathcal{O}_{X}\right)$ is a finitely generated $K$ algebra of dimension $d$. Thus the Iitaka-dimension of $D$ is maximal, and $D$ is called big; see [21. Definitions 2.1.3 and 2.2.1] This means that there exists a number $c>0$ such that $h^{0}\left(X, \mathcal{O}_{X}(k D)\right) \geq c k^{d}$ for $k \gg 0$. Hence from conditions which imply that $S^{k}(\mathcal{G})=\Gamma\left(\mathbb{P}(\mathcal{G}), \mathcal{O}_{\mathbb{P}(\mathcal{G})}(1)\right)$ has few sections, we may derive that $\mathbb{P}\left(\mathcal{G}^{\prime}\right)-\mathbb{P}(\mathcal{G})$ is not affine.

Lemma 4.3. Let $Y$ denote a smooth projective curve over an algebraically closed field and let $\mathcal{T}$ denote a locally free sheaf on $Y$. Suppose that $\bar{\mu}_{\min }(\mathcal{T}) \geq 0$. Then $\mu_{\min }\left(\Gamma^{k}(\mathcal{T})\right) \geq 0$ for $k \geq 0$, where $\Gamma^{k}(\mathcal{T})=\left(S^{k}\left(\mathcal{T}^{\vee}\right)\right)^{\vee}$.

Proof. Note that $S^{k}(\mathcal{T}) \cong\left(S^{k}\left(\mathcal{T}^{\vee}\right)\right)^{\vee}$ is only true in characteristic zero. Assume that there exists a locally free quotient sheaf $\left(S^{k}\left(\mathcal{T}^{\vee}\right)\right)^{\vee} \rightarrow \mathcal{Q} \rightarrow 0$ of negative degree. We find a finite morphism $\varphi: Y^{\prime} \rightarrow Y$ such that we may write $\varphi^{*}(\mathcal{Q})=$ $\mathcal{L}^{k} \otimes \mathcal{N}$, where $\mathcal{L}$ is an invertible sheaf with $\operatorname{deg}(\mathcal{L})<0$ and also $\operatorname{deg}(\mathcal{N})<0$. Due to our assumption we may assume that this is already true on $Y$. We tensor by $\mathcal{L}^{-k}$ and get $\left(S^{k}\left(\mathcal{T}^{\vee}\right)\right)^{\vee} \otimes \mathcal{L}^{-k} \rightarrow \mathcal{N} \rightarrow 0$. But

$$
\left(S^{k}\left(\mathcal{T}^{\vee}\right)\right)^{\vee} \otimes \mathcal{L}^{-k}=\left(S^{k}\left(\mathcal{T}^{\vee}\right) \otimes \mathcal{L}^{k}\right)^{\vee}=\left(S^{k}\left(\mathcal{T}^{\vee} \otimes \mathcal{L}\right)\right)^{\vee}=\left(S^{k}\left(\left(\mathcal{T} \otimes \mathcal{L}^{\vee}\right)^{\vee}\right)\right)^{\vee}
$$


Now $\operatorname{deg}\left(\mathcal{L}^{\vee}\right)>0$, hence $\bar{\mu}_{\min }\left(\mathcal{T} \otimes \mathcal{L}^{\vee}\right)>0$ and therefore $\mathcal{T} \otimes \mathcal{L}^{\vee}$ is ample due to Theorem [2.3, Then due to [10, Theorem 6.6 and Proposition 7.3] it follows that $\left(S^{k}\left(\left(\mathcal{T} \otimes \mathcal{L}^{\vee}\right)^{\vee}\right)\right)^{\vee}$ is ample, buts its quotient sheaf $\mathcal{N}$ is not, since $\operatorname{deg}(\mathcal{N})<0$, which gives a contradiction.

Theorem 4.4. Let the notation be as in Situation 3.1. Suppose that $\bar{\mu}_{\max }(\mathcal{G}) \leq 0$. Then $\mathbb{P}\left(\mathcal{G}^{\prime}\right)-\mathbb{P}(\mathcal{G})$ is not affine.

Proof. From the sequence $0 \rightarrow \mathcal{O}_{Y} \rightarrow \mathcal{G}^{\prime} \rightarrow \mathcal{G} \rightarrow 0$ it follows that $\bar{\mu}_{\text {max }}\left(\mathcal{G}^{\prime}\right) \leq 0$ holds as well, since the image of a mapping $\mathcal{S} \rightarrow \mathcal{G}^{\prime}$, where $\mathcal{S}$ is a semistable sheaf of positive slope, must lie inside the kernel of $\mathcal{G}^{\prime} \rightarrow \mathcal{G}$. Applying Lemma 4.3 to the dual of $\mathcal{G}^{\prime}$, it follows that $\mu_{\max }\left(S^{k}\left(\mathcal{G}^{\prime}\right)\right) \leq 0$.

If moreover $\mu_{\max }\left(S^{k}\left(\mathcal{G}^{\prime}\right)\right)<0$, then $\Gamma\left(Y, S^{k}\left(\mathcal{G}^{\prime}\right)\right)=0$. So we may suppose that $\mu_{\max }\left(S^{k}\left(\mathcal{G}^{\prime}\right)\right)=0$. Then there exists the maximal destabilizing sheaf $0 \rightarrow \mathcal{F}_{k} \rightarrow$ $S^{k}\left(\mathcal{G}^{\prime}\right) \rightarrow S^{k}\left(\mathcal{G}^{\prime}\right) / \mathcal{F}_{k} \rightarrow 0$ such that $\mu\left(\mathcal{F}_{k}\right)=0$ and $\mu_{\max }\left(S^{k}\left(\mathcal{G}^{\prime}\right) / \mathcal{F}_{k}\right)<0$, so that this sheaf again has no global sections $\neq 0$.

We claim that for a semistable locally free sheaf $\mathcal{F}$ of degree zero and rank $s$ the dimension of the global sections is at most $s$ : This is true for invertible sheaves, so we do induction on the rank. If $\mathcal{F}$ has a global section $\neq 0$, then $\mathcal{O}_{Y} \subseteq \mathcal{F}$ is a subsheaf. We consider the saturation $\mathcal{O}_{Y} \subseteq \mathcal{M} \subseteq \mathcal{F}$ so that the cokernel $\mathcal{F} / \mathcal{M}$ is torsion free, hence locally free on the curve (see [18, 1.1]). $\mathcal{M}$ has degree 0 (since $\mathcal{O}_{Y} \subseteq \mathcal{M}$ and since $\mathcal{F}$ is semistable) and therefore we may apply the induction hypothesis to the cokernel.

This gives the estimates $\left(\operatorname{set} r=\operatorname{rk}(\mathcal{G})\right.$ and $r+1=\operatorname{rk}\left(\mathcal{G}^{\prime}\right)$ )

$$
h^{0}\left(S^{k}\left(\mathcal{G}^{\prime}\right)\right) \leq h^{0}\left(\mathcal{F}_{k}\right)+h^{0}\left(S^{k}\left(\mathcal{G}^{\prime}\right) / \mathcal{F}_{k}\right) \leq \operatorname{rk}\left(\mathcal{F}_{k}\right) \leq \operatorname{rk}\left(S^{k}\left(\mathcal{G}^{\prime}\right)\right)=\left(\begin{array}{c}
k+r \\
r
\end{array}\right) .
$$

This is a polynomial with leading coefficient $\frac{1}{r !} k^{r}$, thus it is bounded above by $\leq c k^{r}$ for some $c>0$. Since the dimension of $\mathbb{P}\left(\mathcal{G}^{\prime}\right)$ is $r+1$ and since $\pi_{*} \mathcal{O}_{\mathbb{P}\left(\mathcal{G}^{\prime}\right)}(k)=S^{k}\left(\mathcal{G}^{\prime}\right)$, it follows that $\mathcal{O}_{\mathbb{P}\left(\mathcal{G}^{\prime}\right)}(1)$ is not big and that $\mathbb{P}\left(\mathcal{G}^{\prime}\right)-\mathbb{P}(\mathcal{G})$ is not affine by Remark 4.2 .

Corollary 4.5. Let the notation be as in Situation 3.1. Suppose that $\mathcal{G}$ is strongly semistable and $\mu(\mathcal{G}) \leq 0$. Then $\mathbb{P}\left(\mathcal{G}^{\prime}\right)-\mathbb{P}(\mathcal{G})$ is not affine.

Proof. Since $\mathcal{G}$ is strongly semistable we have $\bar{\mu}_{\max }(\mathcal{G})=\mu(\mathcal{G}) \leq 0$, hence the result follows from Theorem 4.4

Remark 4.6. For an elliptic curve $Y$ one can improve Theorems 3.4 and 4.4 to a complete characterization of affine subsets (in any characteristic). Suppose the notation of Situation [3.1, and let $\mathcal{S}=\mathcal{S}_{1} \oplus \ldots \oplus \mathcal{S}_{s}$ be the decomposition into indecomposable sheaves, $c=\left(c_{j}\right), c_{j} \in H^{1}\left(Y, \mathcal{S}_{j}\right)$. An indecomposable sheaf on an elliptic curve is strongly semistable, hence we have in this situation a much better control on the different notions of slopes. Theorem 3.2 of [4] states that $\mathbb{P}\left(\mathcal{G}^{\prime}\right)-\mathbb{P}(\mathcal{G})$ is affine if and only if there exists $j$ such that $\operatorname{deg}\left(\mathcal{S}_{j}\right)<0$ and $c_{j} \neq 0$. In positive characteristic, the same numerical condition is equivalent to the non-existence of projective curves inside $\mathbb{P}\left(\mathcal{G}^{\prime}\right)-\mathbb{P}(\mathcal{G})$; see [4, Theorem 3.3].

\section{Projective Bundles Corresponding to tight Closure Problems}

In this section we briefly recall some results from [3] on how graded tight closure problems in a graded ring $R$ translate to problems about projective bundles and 
subbundles over Proj $R$. Let $K$ denote an algebraically closed field and let $R$ be a standard $\mathbb{N}$-graded $K$-algebra, that is, $R_{0}=K$ and $R$ is generated by finitely many elements of degree one. Set $Y=\operatorname{Proj} R$. Let $f_{i}$ be homogeneous $R_{+}$-primary elements of $R$ of degree $d_{i}$, that is, the $D_{+}\left(f_{i}\right)$ cover $Y$. Then for $m \in \mathbb{Z}$ we have the exact sequence of locally free sheaves on $Y$,

$$
0 \longrightarrow \mathcal{R}(m) \longrightarrow \mathcal{O}_{Y}\left(m-d_{1}\right) \oplus \ldots \oplus \mathcal{O}_{Y}\left(m-d_{n}\right) \stackrel{\sum f_{i}}{\longrightarrow} \mathcal{O}_{Y}(m) \longrightarrow 0,
$$

and dually

$$
0 \longrightarrow \mathcal{O}_{Y}(-m) \longrightarrow \mathcal{O}_{Y}\left(d_{1}-m\right) \oplus \ldots \oplus \mathcal{O}_{Y}\left(d_{n}-m\right) \longrightarrow \mathcal{F}(-m) \longrightarrow 0 .
$$

$\mathcal{R}(m)$ is the sheaf of relations of total degree $m$ corresponding to the generators $f_{1}, \ldots, f_{n}$. Its rank is $n-1$. From this presenting sequence it follows that

$$
\operatorname{Det}(\mathcal{F}(-m))=\mathcal{O}_{Y}\left(d_{1}+\ldots+d_{n}-(n-1) m\right)
$$

and

$$
\operatorname{deg}(\mathcal{F}(-m))=\left(d_{1}+\ldots+d_{n}-(n-1) m\right) \operatorname{deg}\left(\mathcal{O}_{Y}(1)\right) .
$$

A geometric realization of the vector bundle $V(-m)=\operatorname{Spec} \oplus S^{k}(\mathcal{F}(-m))$ is given by the open subset

$$
V(-m)=D_{+}\left(R_{+}\right) \subset \operatorname{Proj} R\left[T_{1}, \ldots, T_{n}\right] /\left(\sum_{i=1}^{n} f_{i} T_{i}\right),
$$

where $\operatorname{deg}\left(T_{i}\right)=m-d_{i}$.

Now suppose that $f_{0}$ is another homogeneous element in $R$ of degree $m$. The elements $f_{1}, \ldots, f_{n}, f_{0}$ again define a sheaf of relations $\mathcal{R}^{\prime}(m)$ together with a short exact sequence

$$
0 \longrightarrow \mathcal{R}(m) \longrightarrow \mathcal{R}^{\prime}(m) \longrightarrow \mathcal{O}_{Y} \longrightarrow 0
$$

which we call the forcing sequence. The corresponding cohomology class $c \in$ $H^{1}(Y, \mathcal{R}(m)$ ) (the forcing class) is also given by the connecting homomorphism $R_{m} \rightarrow \Gamma\left(Y, \mathcal{O}_{Y}(m)\right) \rightarrow H^{1}(Y, \mathcal{R}(m))$. The dual sequence

$$
0 \longrightarrow \mathcal{O}_{Y} \longrightarrow \mathcal{F}^{\prime}(-m) \longrightarrow \mathcal{F}(-m) \longrightarrow 0
$$

yields a closed subbundle $\mathbb{P}(\mathcal{F}(-m)) \subset \mathbb{P}\left(\mathcal{F}^{\prime}(-m)\right.$ ) (we will skip the number $m$ in this expression and write $\mathbb{P}(\mathcal{F})$ ).

The basic fact is that the complement $\mathbb{P}\left(\mathcal{F}^{\prime}\right)-\mathbb{P}(\mathcal{F})$ is isomorphic to the Proj of the so-called forcing algebra $R\left[T_{1}, \ldots, T_{n}\right] /\left(f_{1} T_{1}+\ldots+f_{n} T_{n}+f_{0}\right)$ (suitable graded). See 14 for forcing algebras and how the tight closure of an ideal is expressed in terms of them. The containment of a homogeneous element in the ideal, in the tight closure and in the plus closure of the ideal, is expressed in terms of these projective bundles in the following way. Note that in characteristic zero we work with the notion of solid closure.

Lemma 5.1. In the described situation the following are equivalent:

(i) $f_{0} \in\left(f_{1}, \ldots, f_{n}\right)$.

(ii) There exists a section $Y \rightarrow \mathbb{P}\left(\mathcal{F}^{\prime}\right)$ disjoined to $\mathbb{P}(\mathcal{F}) \subset \mathbb{P}\left(\mathcal{F}^{\prime}\right)$.

(iii) The forcing sequence $0 \rightarrow \mathcal{R}(m) \rightarrow \mathcal{R}^{\prime}(m) \rightarrow \mathcal{O}_{Y} \rightarrow 0$ splits.

(iv) The corresponding cohomological class in $H^{1}(Y, \mathcal{R}(m))$ vanishes.

Proof. See [3, Lemma 3.7]. 
Proposition 5.2. Let $R$ be a normal standard-graded $K$-algebra of dimension 2, let $f_{1}, \ldots, f_{n} \in R$ be $R_{+}$-primary homogeneous elements and let $f_{0}$ be another homogeneous element. Then $f_{0} \in\left(f_{1}, \ldots, f_{n}\right)^{*}$ if and only if $\mathbb{P}\left(\mathcal{F}^{\prime}\right)-\mathbb{P}(\mathcal{F})$ is not affine.

Furthermore, if the characteristic of $K$ is positive, the following are equivalent:

(i) $f_{0} \in\left(f_{1}, \ldots, f_{n}\right)^{+\mathrm{gr}}$, that is, there exists a finite graded extension $R \subseteq R^{\prime}$ such that $f_{0} \in\left(f_{1}, \ldots, f_{n}\right) R^{\prime}$.

(ii) There exists a smooth projective curve $Z$ and a finite surjective morphism $g: Z \rightarrow Y$ such that the pull back $g^{*} \mathbb{P}\left(\mathcal{F}^{\prime}\right)$ has a section not meeting $g^{*} \mathbb{P}(\mathcal{F})$.

(iii) There exists a curve $Z \subset \mathbb{P}\left(\mathcal{F}^{\prime}\right)$ which does not intersect $\mathbb{P}(\mathcal{F})$.

Proof. See [3, Lemmata 3.9 and 3.10].

\section{Applichtions to tight Closure: InClusion bounds}

We shall now apply the results of the previous sections to tight closure problems. We fix the following situation.

Situation 6.1. Let $K$ denote a field with algebraic closure $\bar{K}$ and let $R$ denote a two-dimensional standard-graded $K$-algebra such that $R_{\bar{K}}$ is a normal domain. Let $Y=\operatorname{Proj} R$ denote the corresponding smooth projective curve over $\bar{K}$, let $g$ denote its genus and let $\delta=\operatorname{deg}(Y)=\operatorname{deg}\left(\mathcal{O}_{Y}(1)\right)$ denote the degree of the very ample invertible sheaf $\mathcal{O}_{Y}(1)$ on $Y$. Let $f_{1}, \ldots, f_{n} \in R$ be homogeneous $R_{+}$-primary elements of degree $d_{i}$. Let $\mathcal{R}(m)$ be the sheaf of relations of total degree $m$ on $Y$ and let $\mathcal{F}(-m)$ be its dual sheaf.

Let $f_{0}$ denote another homogeneous element of degree $m$, let $\mathcal{R}^{\prime}(m)$ be the sheaf of relations for the elements $f_{1}, \ldots, f_{n}, f_{0}$ of total degree $m$ and let

$$
0 \longrightarrow \mathcal{R}(m) \longrightarrow \mathcal{R}^{\prime}(m) \longrightarrow \mathcal{O}_{Y} \longrightarrow 0
$$

be the corresponding extension and let $c \in H^{1}(Y, \mathcal{R}(m))$ be the corresponding forcing class defined by $f_{0}$. The corresponding surjection $\mathcal{F}^{\prime}(-m) \rightarrow \mathcal{F}(-m) \rightarrow 0$ yields the embedding $\mathbb{P}(\mathcal{F}(-m)) \subset \mathbb{P}\left(\mathcal{F}^{\prime}(-m)\right)$.

Definition 6.2. Let the notation be as in Situation 6.1. Then we set

$$
\mu_{\max }\left(f_{1}, \ldots, f_{n}\right):=\mu_{\max }(\mathcal{F}(0))
$$

and also for $\mu, \mu_{\min }, \bar{\mu}_{\max }$ and $\bar{\mu}_{\min }$.

Remark 6.3. Note that we always consider for ideal generators $f_{1}, \ldots, f_{n} \in R$ the slope of the relation bundle after replacing $K$ by $\bar{K}$. Since changing the base field does not affect the affineness of open subsets, it does not affect solid closure.

The slope of $\mathcal{F}(0)$ is - due to the presenting sequence for $\mathcal{F}(0)$ - given by

$$
\mu(\mathcal{F}(0))=\frac{d_{1}+\ldots+d_{n}}{n-1} \delta .
$$

Therefore we get the estimates

$$
\mu_{\min }\left(f_{1}, \ldots, f_{n}\right) \leq \frac{d_{1}+\ldots+d_{n}}{n-1} \delta \leq \mu_{\max }\left(f_{1}, \ldots, f_{n}\right) .
$$

Equality holds if and only if $\mathcal{F}(0)$ is semistable. Furthermore we have

$$
\mu(\mathcal{F}(-m))=\mu\left(\mathcal{F}(0) \otimes \mathcal{O}_{Y}(-m)\right)=\mu(\mathcal{F}(0))-m \delta=\left(\frac{d_{1}+\ldots+d_{n}}{n-1}-m\right) \delta,
$$


and the same rule holds for $\mu_{\max }$, etc. If $f_{0}$ is another homogeneous element of degree $m$, then the number

$$
\operatorname{deg}(\mathcal{F}(-m))=\operatorname{deg}\left(\mathcal{F}^{\prime}(-m)\right)=\left(d_{1}+\ldots+d_{n}-(n-1) m\right) \delta
$$

is also the top self-intersection number of the forcing subbundle $\mathbb{P}(\mathcal{F}) \subseteq \mathbb{P}\left(\mathcal{F}^{\prime}\right)$.

From the conditions in section 4 we derive the following numerical condition that elements of sufficiently high degree must belong to the tight closure.

Theorem 6.4. Let the notation be as in Situation 6.1 If

$$
\operatorname{deg}\left(f_{0}\right) \geq \frac{1}{\delta} \bar{\mu}_{\max }\left(f_{1}, \ldots, f_{n}\right),
$$

then $f_{0} \in\left(f_{1}, \ldots, f_{n}\right)^{*}$.

Proof. Let $m=\operatorname{deg}\left(f_{0}\right)$. The condition means that

$$
\bar{\mu}_{\text {max }}(\mathcal{F}(-m))=\bar{\mu}_{\max }\left(f_{1}, \ldots, f_{n}\right)-m \delta \leq 0 .
$$

Hence the result follows from Theorem 4.4 and Proposition 5.2 .

Remark 6.5. This numerical criterion generalizes the corresponding statement for cones over elliptic curves proved in 4, Corollary 4.9]. On an elliptic curve we have the equality $\bar{\mu}_{\max }(\mathcal{F}(0))=\mu_{\max }(\mathcal{F}(0))=\max _{j}\left(\mu\left(\mathcal{F}_{j}\right)\right)$, where $\mathcal{F}(0)=\bigoplus_{j} \mathcal{F}_{j}$ is the decomposition into indecomposable sheaves.

To obtain criteria for tight closure membership we need bounds from above for $\bar{\mu}_{\max }\left(f_{1}, \ldots, f_{n}\right)$. The next proposition gives a general bound for $\bar{\mu}_{\max }$. We will give a much better bound in section 9 for the case $n=3$ under the condition that the sheaf of relations is indecomposable.

Proposition 6.6. Let the notation be as in Situation 6.1. Suppose that the degrees are ordered $1 \leq d_{1} \leq d_{2} \leq \ldots \leq d_{n}$. Let $\mathcal{E}=\bigoplus \mathcal{O}_{Y}\left(d_{i}\right)$ and let $0 \rightarrow \mathcal{O}_{Y} \rightarrow \mathcal{E} \rightarrow$ $\mathcal{F}(0) \rightarrow 0$ be the presenting sequence for $\mathcal{F}(0)$. Then we have the estimate

$$
\mu_{\max }\left(f_{1}, \ldots, f_{n}\right) \leq \delta \cdot\left(d_{n-1}+d_{n}\right) .
$$

The same is true for $\bar{\mu}_{\max }$.

Proof. Set $\mathcal{F}=\mathcal{F}(0)$. Corollary 2.6 together with the inequality $\rho_{s}(\mathcal{F}) \geq \rho_{s}(\mathcal{E})$ yields

$$
\mu_{\max }(\mathcal{F}) \leq \max _{s=0, \ldots, n-2} \frac{\operatorname{deg}(\mathcal{F})-\rho_{s}(\mathcal{F})}{n-1-s} \leq \max _{s=0, \ldots, n-2} \frac{\operatorname{deg}(\mathcal{E})-\rho_{s}(\mathcal{E})}{n-1-s} .
$$

We claim that $\rho_{s}(\mathcal{E})=\delta\left(d_{1}+\ldots+d_{s}\right)$. Since $\mathcal{O}_{Y}\left(d_{1}\right) \oplus \ldots \oplus \mathcal{O}_{Y}\left(d_{s}\right)$ is a quotient sheaf of rank $s$, the estimate $\leq$ is clear. For the other estimate we first consider the case $s=1$, so suppose that $\mathcal{Q}$ is an invertible sheaf. If $\mathcal{Q}$ is a quotient of $\mathcal{E}$, then $\operatorname{Hom}\left(\mathcal{O}_{Y}\left(d_{i}\right), \mathcal{Q}\right)=H^{0}\left(Y, \mathcal{O}_{Y}\left(-d_{i}\right) \otimes \mathcal{Q}\right) \neq 0$ for at least one $i$. Therefore $\operatorname{deg}(\mathcal{Q}) \geq \delta \min _{i} d_{i}=\delta d_{1}$.

Now suppose that $\mathcal{Q}$ is a locally free quotient of $\mathcal{E}$ of rank $s$. Then we have a surjection

$$
\bigoplus_{i_{1}<\ldots<i_{s}} \mathcal{O}_{Y}\left(d_{i_{1}}\right) \otimes \ldots \otimes \mathcal{O}_{Y}\left(d_{i_{s}}\right) \cong \bigwedge^{s} \mathcal{E} \longrightarrow \bigwedge^{s} \mathcal{Q}=\operatorname{det} \mathcal{Q}
$$

Due to the case $s=1$ we know $\operatorname{deg}(\mathcal{Q}) \geq \delta\left(d_{1}+\ldots+d_{s}\right)$, which proves the claim. 
Thus we have the estimate

$$
\mu_{\max }(\mathcal{F}) \leq \max _{s=0, \ldots, n-2} \frac{\operatorname{deg}(\mathcal{E})-\rho_{s}(\mathcal{E})}{n-1-s}=\max _{s=0, \ldots, n-2} \delta \frac{d_{s+1}+\ldots+d_{n}}{n-1-s} .
$$

Here the term for $s=n-2$, which is $\delta\left(d_{n-1}+d_{n}\right)$, is maximal.

If $\varphi: Y^{\prime} \rightarrow Y$ is a finite morphism, then the situation is preserved under the pull-back (even if $\varphi^{*} \mathcal{O}_{Y}(1)$ is not very ample anymore). Then $\mu_{\max }\left(\varphi^{*}(\mathcal{F})\right) \leq$ $(\delta \cdot \operatorname{deg}(\varphi))\left(d_{n-1}+d_{n}\right)$ and hence the inequality also holds for $\bar{\mu}_{\max }$.

Corollary 6.7. Let the notation be as in Situation 6.1. Suppose that the degrees are ordered $1 \leq d_{1} \leq d_{2} \leq \ldots \leq d_{n}$. Then for $m \geq d_{n-1}+d_{n}$ we have the inclusion

$$
R_{m} \subseteq\left(f_{1}, \ldots, f_{n}\right)^{*} .
$$

Proof. This follows from Proposition 6.6 and Theorem 6.4

Remark 6.8. This corollary is for the two-dimensional case, a somewhat better estimate than the estimate $2 \max _{i}\left(d_{i}\right)$ (and $d_{1}+\ldots+d_{n}$ ) found by K. Smith; see 26. Proposition 3.1 and Proposition 3.3]. Smith's estimate for $R_{+}$-primary ideals is however true in every dimension and also holds for the plus closure in positive characteristic.

Remark 6.9. An estimate from below for $\mu_{\max }$ is $\mu_{\max }(\mathcal{F}) \geq \delta \cdot \max _{i}\left(d_{i}\right)$. To see this consider again the sequence $0 \rightarrow \mathcal{O}_{Y} \rightarrow \bigoplus_{i} \mathcal{O}_{Y}\left(d_{i}\right) \rightarrow \mathcal{F} \rightarrow 0$. If $\mathcal{O}_{Y}\left(d_{i}\right) \rightarrow \mathcal{F}$ is the zero map for one $i$, then $\mathcal{O}_{Y} \cong \mathcal{O}_{Y}\left(d_{i}\right), d_{i}=0$ and $f_{i}$ is a unit, and the statement is clear from $\mathcal{F} \cong \bigoplus_{j \neq i} \mathcal{O}_{Y}\left(d_{j}\right)$. Otherwise $\mathcal{O}_{Y}\left(d_{i}\right) \rightarrow \mathcal{F}$ is not the zero map, and then $\mu_{\max }(\mathcal{F}) \geq \mu\left(\mathcal{O}_{Y}\left(d_{i}\right)\right)=\delta d_{i}$ for all $i$.

\section{Applications to tight Closure: Exclusion bounds}

We are now looking for degree bounds $a$ such that if $\operatorname{deg}\left(f_{0}\right)<a$, then $f_{0} \in$ $\left(f_{1}, \ldots, f_{n}\right)^{*}$ if and only if $f_{0} \in\left(f_{1}, \ldots, f_{n}\right)$. So below the degree bound an element $f_{0}$ is excluded from the tight closure with the exception that it belongs to the ideal itself.

The theorems in this and the next section hold either in characteristic zero or in positive characteristic $p$ under the condition that $p \gg 0$. To make sense of this statement we have to suppose that everything is given relative to a base scheme such that the generic fiber has characteristic zero and the special fibers have positive characteristic. For this we fix the following situation; see also [17, Definition 3.3] and in particular the appendix of Hochster in [15] for this setting.

Situation 7.1. Let $D$ denote a finitely generated normal $\mathbb{Z}$-domain of dimension one. Let $S$ denote a standard-graded flat $D$-algebra such that for all $\mathfrak{p} \in \operatorname{Spec} D$ the algebras $S_{\kappa(\mathfrak{p})}=S \otimes_{D} \kappa(\mathfrak{p})$ are two-dimensional geometrically normal standardgraded $\kappa(\mathfrak{p})$-algebras (so that $S_{\bar{\kappa}(\mathfrak{p})}$ are normal domains). For $\mathfrak{p}=0$ this is an algebra over the quotient field $Q(D)$ of characteristic zero, and for a maximal ideal $\mathfrak{p}$ the algebra $S_{\kappa(\mathfrak{p})}$ is an algebra over the finite field $\kappa(\mathfrak{p})=D / \mathfrak{p}$ of positive characteristic.

We suppose that we have $S_{+}$-primary homogeneous elements $f_{1}, \ldots, f_{n} \in S$ of degree $d_{i}$ and another homogeneous element $f_{0}$. Let $B$ denote the forcing algebra over $S$ for this data and let $U=D\left(S_{+}\right) \subseteq$ Spec $B$. These elements yield 
homogeneous forcing data for every $S_{\kappa(\mathfrak{p})}$, and $B_{\kappa(\mathfrak{p})}=B \otimes_{D} \kappa(\mathfrak{p})$ is the corresponding forcing algebra. For every prime ideal $\mathfrak{p} \in \operatorname{Spec} D$ the affineness of $U_{\mathfrak{p}}=U_{\kappa(\mathfrak{p})}=U \cap \operatorname{Spec} B_{\kappa(\mathfrak{p})}$ is equivalent to $f_{0} \notin\left(f_{1}, \ldots, f_{n}\right)^{*}$ in $S_{\kappa(\mathfrak{p})}$.

We denote by $Y=\operatorname{Proj} S$ the smooth projective (relative) curve over Spec $D$ and by $\delta$ the common degree of the curves $Y_{\mathfrak{p}}, \mathfrak{p} \in \operatorname{Spec} D$. We denote by $\mathcal{R}(m)$ the sheaf of relations on $Y$ and by $\mathcal{F}(-m)$ its dual sheaf, and we denote the restrictions to $Y_{\mathfrak{p}}$ by $\mathcal{R}_{\mathfrak{p}}(m)$ and $\mathcal{F}_{\mathfrak{p}}(-m)$. The different notions of slopes and of semistability refer always to $\mathcal{F}_{\bar{\kappa}(\mathfrak{p})}$ on $Y_{\bar{\kappa}(\mathfrak{p})}$. The element $f_{0}$ yields an extension $0 \rightarrow \mathcal{R}(m) \rightarrow$ $\mathcal{R}^{\prime}(m) \rightarrow \mathcal{O}_{Y} \rightarrow 0$ and a subbundle $\mathbb{P}(\mathcal{F}) \subset \mathbb{P}\left(\mathcal{F}^{\prime}\right)$, which induces the projective subbundle $\mathbb{P}\left(\mathcal{F}_{\mathfrak{p}}\right) \subset \mathbb{P}\left(\mathcal{F}_{\mathfrak{p}}^{\prime}\right)$ on every curve for every $\mathfrak{p} \in \operatorname{Spec} D$.

Remark 7.2. We will apply several times the following conclusion: let $D \subseteq B$ denote Noetherian domains and let $U=D(\mathfrak{a}) \subseteq$ Spec $B$ denote an open subset, $\mathfrak{a}=\left(a_{1}, \ldots, a_{k}\right)$. Suppose that $U_{\eta}=U \cap \operatorname{Spec}\left(B \otimes_{D} \kappa(\eta)\right)$ is affine, where $\eta$ denotes the generic point of Spec $D$. This means that there exist rational functions $q_{j} \in \Gamma\left(U_{\eta}, \mathcal{O}_{\eta}\right)$ such that $\sum q_{j} a_{j}=1$. We find a common denominator $0 \neq g \in D$ such that these functions $q_{j}$ are defined on $U \cap D(g) \subseteq \operatorname{Spec} B$, hence also $U \cap D(g)$ is affine. This means that after shrinking $D$ (i.e. replacing $\operatorname{Spec} D$ by $\operatorname{Spec} D_{g}$ ) we may assume that $U$ itself is affine. Hence for every $P \in \operatorname{Spec} D$ the fibers $U_{\kappa(P)}$ are affine.

Suppose in Situation 7.1 that $f_{0} \notin\left(f_{1}, \ldots, f_{n}\right)^{*}$ holds over the generic point $\eta \in \operatorname{Spec} D$. This means that the open subset $U_{\eta}$ is affine. Then after shrinking $D$ we may assume that $U$ is affine, hence that every fiber $U_{\kappa(\mathfrak{p})}$ is affine. This means that $f_{0} \notin\left(f_{1}, \ldots, f_{n}\right)^{*}$ holds in $S_{\kappa(\mathfrak{p})}$ for all $\mathfrak{p} \in \operatorname{Spec} D$ (or for almost all $\mathfrak{p} \in \operatorname{Spec} D$ for the old $D)$. In this case we say briefly that $f_{0} \notin\left(f_{1}, \ldots, f_{n}\right)^{*}$ holds for $p \gg 0$.

Theorem 7.3. Let the notation be as in Situations 6.1 and in 7.1. Suppose that the characteristic of $K$ is 0 or $p \gg 0$. If $\operatorname{deg}\left(f_{0}\right)<\frac{1}{\delta} \mu_{\min }\left(f_{1}, \ldots, f_{n}\right)$ $\left(=\frac{1}{\delta} \mu_{\min }\left(\mathcal{F}_{\eta}(0)\right)\right)$, then $f_{0} \in\left(f_{1}, \ldots, f_{n}\right)^{*}$ if and only if $f_{0} \in\left(f_{1}, \ldots, f_{n}\right)$.

Proof. Let $m=\operatorname{deg}\left(f_{0}\right)$. Suppose first that the characteristic is zero. We may assume that $K$ is algebraically closed. The condition means that $\mu_{\min }(\mathcal{F}(-m))=$ $\mu_{\min }\left(f_{1}, \ldots, f_{n}\right)-m \delta>0$, hence $\mathcal{F}(-m)$ is ample due to Theorem2.1. Suppose that $f_{0} \notin\left(f_{1}, \ldots, f_{n}\right)$. This means by Lemma 5.1 that the corresponding forcing class is $c \neq 0$. Hence $\mathbb{P}\left(\mathcal{F}^{\prime}\right)-\mathbb{P}(\mathcal{F})$ is affine due to Corollary 3.2 and $f_{0} \notin\left(f_{1}, \ldots, f_{n}\right)^{*}$ due to Proposition 5.2

Now suppose the relative Situation [7.1. Note that the slope condition is imposed on the generic fiber. We have to show that $f_{0} \notin\left(f_{1}, \ldots, f_{n}\right)$ implies $f_{0} \notin$ $\left(f_{1}, \ldots, f_{n}\right)^{*}$ for almost all $\mathfrak{p} \in \operatorname{Spec} D$. From $f_{0} \notin\left(f_{1}, \ldots, f_{n}\right)$ in $S_{\kappa(\mathfrak{p})}$ it follows that $f_{0} \notin\left(f_{1}, \ldots, f_{n}\right)$ in $S$ and by shrinking $D$ we may assume that $f_{0} \notin\left(f_{1}, \ldots, f_{n}\right)$ in $S_{Q(D)}$. From the case of characteristic zero we know that $U_{\eta}$ is affine and the result follows from Remark 7.2 .

From the bounds proved in Theorem 6.4 and in Theorem 7.3 it is easy to derive the following result of Huneke and Smith (see [17, Theorem 5.11]).

Corollary 7.4. Let the notation be as in Situation 6.1 (or Situation [7.1). Suppose that the characteristic of $K$ is 0 or $p \gg 0$. Suppose that the projective dimension of $R /\left(f_{1}, \ldots, f_{n}\right)$ is 2 or equivalently that the sheaf of relations $\mathcal{R}(0) \cong \mathcal{O}_{Y}\left(-a_{1}\right) \oplus$ 
$\ldots \oplus \mathcal{O}_{Y}\left(-a_{r}\right)$. Let $a=\max \left\{a_{i}\right\}$ and $b=\min \left\{a_{i}\right\}$. Then

$$
R_{\geq a} \subseteq\left(f_{1}, \ldots, f_{n}\right)^{*} \text { and }\left(f_{1}, \ldots, f_{n}\right)^{*} \subseteq\left(f_{1}, \ldots, f_{n}\right)+R_{\geq b} .
$$

Proof. Whenever $\mathcal{F}(0)$ is a direct sum of invertible sheaves $\mathcal{L}_{j}$ we have $\bar{\mu}_{\text {max }}(\mathcal{F}(0))=$ $\max _{j} \operatorname{deg}\left(\mathcal{L}_{j}\right)$ and $\bar{\mu}_{\min }(\mathcal{F}(0))=\min _{j} \operatorname{deg}\left(\mathcal{L}_{j}\right)$. So for $\mathcal{F}(0)=\mathcal{O}_{Y}\left(a_{1}\right) \oplus \ldots \oplus$ $\mathcal{O}_{Y}\left(a_{r}\right)$ we find that $\bar{\mu}_{\max }(\mathcal{F}(0))=a \delta$ and $\bar{\mu}_{\min }(\mathcal{F}(0))=b \delta$, so the result follows from Theorems 6.4 and 7.3 .

If the sheaf of relations on the projective curve splits into invertible sheaves as in the previous Corollary [7.4 it is easy to give a numerical criterion for tight closure.

Theorem 7.5. Let the notation be as in Situation 6.1 (or Situation 7.1). Suppose that we have a decomposition $\mathcal{R}(0)=\mathcal{L}_{1} \oplus \ldots \oplus \mathcal{L}_{s}$, where $\mathcal{L}_{j}$ are invertible sheaves of degree $\mu_{j}$. Let $m=\operatorname{deg}\left(f_{0}\right)$ and let $c \in H^{1}(Y, \mathcal{R}(m))$ be the forcing class with components $c_{j} \in H^{1}\left(Y, \mathcal{L}_{j} \otimes \mathcal{O}_{Y}(m)\right)$. Suppose that the characteristic is zero or $p \gg 0$. Then $f_{0} \in\left(f_{1}, \ldots, f_{n}\right)^{*}$ if and only if $\operatorname{deg}\left(\mathcal{L}_{j}\right)+m \delta \geq 0$ or $c_{j}=0$ holds for all $1 \leq j \leq s$.

Proof. Suppose first that $f_{0} \in\left(f_{1}, \ldots, f_{n}\right)^{*}$ holds and consider to the contrary that there exists $j$ such that $\operatorname{deg}\left(\mathcal{L}_{j}\right)+m \delta<0$ and $c_{j} \neq 0$. Then Corollary 3.6 together with Remark 3.7 yields the contradiction $f_{0} \notin\left(f_{1}, \ldots, f_{n}\right)^{*}$.

For the other direction we consider the direct summand

$$
\mathcal{S}=\bigoplus_{\operatorname{deg}\left(\mathcal{L}_{j}(m)\right) \geq 0} \mathcal{L}_{j}(m) \subseteq \mathcal{R}(m) .
$$

Then all non-zero components of the forcing class $c$ belong to $\mathcal{S}$, so this class comes from and goes to a cohomology class in $H^{1}(Y, \mathcal{S})$. Hence we know by Lemma 3.3 that the affineness of $\mathbb{P}\left(\left(\mathcal{S}^{\prime}\right)^{\vee}\right)-\mathbb{P}\left(\mathcal{S}^{\vee}\right)$ is equivalent to the affineness of $\mathbb{P}\left(\mathcal{F}^{\prime}\right)-\mathbb{P}(\mathcal{F})$. Since the degree of every invertible summand sheaf of $\mathcal{S}$ is non-negative, we know that $\bar{\mu}_{\min }(\mathcal{S}) \geq 0$ and then $\bar{\mu}_{\max }\left(\mathcal{S}^{\vee}\right) \leq 0$; therefore $\mathbb{P}\left(\left(\mathcal{S}^{\prime}\right)^{\vee}\right)-\mathbb{P}\left(\mathcal{S}^{\vee}\right)$ is not affine due to Theorem 4.4

Remark 7.6. The situation of Theorem 7.5 holds for every primary homogeneous ideal in $K[x, y]$ (due to the splitting theorem of Grothendieck; see [24, Theorem 2.1.1]), but for a polynomial ring the computation of tight closure does not make many problems, so Theorem 7.5 gives help that we do not need in this case. However, the splitting situation also holds if $I \subseteq R$ is the extended ideal $I=J R$ of an ideal $J \subseteq K[x, y] \subset R$, and in this case it is also useful for computations; see Example 9.7 below. There is also a version of Theorem 7.5 if the sheaf of relations splits into a direct sum of (strongly) semistable sheaves.

Remark 7.7. Let the notation be as in Situation 6.1. From the sequence

$$
0 \longrightarrow \mathcal{O}_{Y} \longrightarrow \mathcal{O}_{Y}\left(d_{1}\right) \oplus \ldots \oplus \mathcal{O}_{Y}\left(d_{n}\right) \longrightarrow \mathcal{F} \longrightarrow 0
$$

we also get the estimate

$$
\bar{\mu}_{\min }(\mathcal{F}) \geq \bar{\mu}_{\min }\left(\bigoplus_{i} \mathcal{O}_{Y}\left(d_{i}\right)\right)=\min _{i} \mu\left(\mathcal{O}_{Y}\left(d_{i}\right)\right)=\delta \min _{i}\left(d_{i}\right) .
$$

So if $f_{0} \neq 0$ and $\operatorname{deg}\left(f_{0}\right)<\min _{i}\left(d_{i}\right)$, then $f_{0} \notin\left(f_{1}, \ldots, f_{n}\right)$ and due to Theorem 7.3 also $f_{0} \notin\left(f_{1}, \ldots, f_{n}\right)^{*}$.

The minimal slope in the generic point also gives a bound for the minimal slope in positive characteristic for $p \gg 0$. The same is true for the maximal slope. 
Proposition 7.8. Suppose the relative Situation 7.1, let $Y=\operatorname{Proj} S \rightarrow$ Spec $D$ denote the smooth projective curve of relative dimension one and suppose that the generic curve $Y_{\eta}=Y_{Q(D)}$ has at least one $Q(D)$-rational point. Let $\mu_{\min }\left(\mathcal{F}_{\eta}(-m)\right)$ denote the minimal slope and let $\mu_{\max }\left(\mathcal{F}_{\eta}(-m)\right)$ denote the maximal slope on $Y_{\bar{\kappa}(\eta)}$. Then for $p \gg 0$ we have the bounds

$$
\bar{\mu}_{\min }(\mathcal{F}(-m))>\left\lceil\mu_{\min }\left(\mathcal{F}_{\eta}(-m)\right)\right\rceil-1
$$

and

$$
\bar{\mu}_{\max }(\mathcal{F}(-m))<\left\lfloor\mu_{\max }\left(\mathcal{F}_{\eta}(-m)\right)\right\rfloor+1 .
$$

Proof. First we may assume by shrinking $D$ that there exists a section for the relative curve $Y$. Hence there exists an invertible sheaf $\mathcal{M}$ on $Y$ such that the degree of $\mathcal{M}$ on every fiber $Y_{\mathfrak{p}}$ is one. Let $\mathcal{L}$ denote an invertible sheaf on $Y$ which has on every fiber $Y_{\mathfrak{p}}$ the degree $-\left\lceil\mu_{\min }\left(\mathcal{F}_{\eta}(-m)\right)\right\rceil+1$.

Then $\mu_{\min }\left(\mathcal{F}_{\eta}(-m) \otimes \mathcal{L}_{\eta}\right)=\mu_{\min }\left(\mathcal{F}_{\eta}(-m)\right)-\left\lceil\mu_{\min }\left(\mathcal{F}_{\eta}(-m)\right)\right\rceil+1>0$ and hence $\mathcal{F}_{\eta}(-m) \otimes \mathcal{L}_{\eta}$ is ample on $Y_{\eta}$ due to Theorem 2.1. Therefore $\mathcal{F}_{\mathfrak{p}}(-m) \otimes \mathcal{L}_{\mathfrak{p}}$ is ample on $Y_{\mathfrak{p}}$ for $p \gg 0$, since ampleness is an open property (see [8, Théorème 4.7.1] or [21, Theorem 1.2.13]; since we need here only the generic open property, we can also use 7.2 together with [7, Théorème 4.5.2]). This means again by Theorem 2.3 that $\bar{\mu}_{\min }\left(\mathcal{F}_{\mathfrak{p}}(-m) \otimes \mathcal{L}_{\mathfrak{p}}\right)>0$ or that

$$
\bar{\mu}_{\text {min }}\left(\mathcal{F}_{\mathfrak{p}}(-m)\right)>-\operatorname{deg}\left(\mathcal{L}_{\mathfrak{p}}\right)=\left\lceil\mu_{\min }\left(\mathcal{F}_{\eta}(-m)\right)\right\rceil-1 .
$$

This gives the first result. The second statement follows by applying the first statement to $\mathcal{R}(m)$,

$$
\begin{aligned}
\bar{\mu}_{\max }(\mathcal{F}(-m)) & =-\bar{\mu}_{\min }(\mathcal{R}(m)) \\
& <-\left(\left\lceil\mu_{\min }\left(\mathcal{R}_{\eta}(m)\right)\right\rceil-1\right) \\
& =\left\lfloor-\mu_{\min }\left(\mathcal{R}_{\eta}(m)\right)\right\rfloor+1 \\
& =\left\lfloor\mu_{\max }\left(\mathcal{F}_{\eta}(-m)\right\rfloor+1 .\right.
\end{aligned}
$$

\section{Applichtions to tight Closure: Vanishing theorems}

The inclusion bound in Theorem 6.4 and the exclusion bound in Theorem 7.3 coincide if the sheaf of relations is semistable. This gives a new class of vanishing type theorems in dimension two and generalizes the vanishing theorem for parameter ideals; see [17] and Corollary [8.6] below (the name vanishing is due to the fact that it is related to the Kodaira Vanishing Theorem). We first give the formulation in zero characteristic.

Theorem 8.1. Let the notation be as in Situation 6.1 and suppose that the characteristic of $K$ is zero. Set $k=\left\lceil\frac{d_{1}+\ldots+d_{n}}{n-1}\right\rceil$. Suppose that the sheaf of relations $\mathcal{R}(m)$ for ideal generators $f_{1}, \ldots, f_{n}$ is semistable. Then

$$
\left(f_{1}, \ldots, f_{n}\right)^{*}=\left(f_{1}, \ldots, f_{n}\right)+R_{\geq k} .
$$

Proof. Let $f_{0} \in R$ be homogeneous of degree $m$. Suppose first that $m \geq k$. Then

$$
m \geq \frac{d_{1}+\ldots+d_{n}}{n-1}=\frac{\mu(\mathcal{F}(0))}{\delta}=\frac{\mu_{\max }\left(f_{1}, \ldots, f_{n}\right)}{\delta} .
$$

Hence the numerical condition in Theorem 6.4 is fulfilled, thus $f_{0} \in\left(f_{1}, \ldots, f_{n}\right)^{*}$.

Suppose now that $m<k$. Then $m<\frac{d_{1}+\ldots+d_{n}}{n-1}=\frac{\mu_{\min }\left(f_{1}, \ldots, f_{n}\right)}{\delta}$ and Theorem 7.3 gives the result. 
Suppose that in the relative setting (Situation [.1) the sheaf of relations is semistable in the generic point, so that the vanishing Theorem 8.1 holds in the generic point. What can we say about the behavior in positive characteristic? We know by $[22, \S 5]$ that $\mathcal{F}_{\mathfrak{p}}$ is semistable on an open non-empty subset of Spec $D$. However, for strongly semistable we have to take into account the following problem of Miyaoka (see also [27]).

Remark 8.2. Miyaoka states in [22, Problem 5.4] the following problem: suppose that $C$ is a relative (smooth projective) curve over a (say) $\mathbb{Z}$-algebra $D$ of finite type and assume that a locally free sheaf $\mathcal{F}$ is semistable in the generic fiber (characteristic zero). Let $S$ be the set of points $P \in \operatorname{Spec} D$ of positive characteristic such that $\mathcal{F} \mid C_{P}$ is strongly semistable. Is $S$ dense in Spec $D$ ?

Therefore we may not expect that semistability in the generic point implies a vanishing theorem for $p \gg 0$ without further conditions. It implies however that the bounds are quite near to the expected number $\left(d_{1}+\ldots+d_{n}\right) /(n-1)$.

Corollary 8.3. Suppose Situation 7.1 and suppose that the sheaf of relations is semistable over the generic point. Let $m=\operatorname{deg}\left(f_{0}\right)$. Then the following hold for $p \gg 0$ :

(i) If $m \geq \frac{d_{1}+\ldots+d_{n}}{n-1}+\frac{1}{\delta}$, then $f_{0} \in\left(f_{1}, \ldots, f_{n}\right)^{*}$.

(ii) If $m \leq \frac{d_{1}+\ldots+d_{n}}{n-1}-\frac{1}{\delta}$, then $f_{0} \in\left(f_{1}, \ldots, f_{n}\right)^{*}$ if and only if $f_{0} \in\left(f_{1}, \ldots, f_{n}\right)$.

Proof. For (i) we have the estimates

$$
\begin{aligned}
\delta m & \geq \delta \frac{d_{1}+\ldots+d_{n}}{n-1}+1 \\
& \geq\left\lfloor\delta \frac{d_{1}+\ldots+d_{n}}{n-1}\right\rfloor+1 \\
& =\left\lfloor\mu_{\max }\left(\mathcal{F}_{\eta}\right)\right\rfloor+1 \\
& >\bar{\mu}_{\max }(\mathcal{F}),
\end{aligned}
$$

where the last estimate follows from Proposition 7.8. The statement follows from Theorem 6.4.

(ii) follows by similar estimates from Theorem 7.3

The previous corollary shows that the inclusion and exclusion bounds are very near to $\left(d_{1}+\ldots+d_{n}\right) /(n-1)$. If this number is not an integer and if the degree of the curve is big enough, then we also get a vanishing theorem from Corollary 8.3 In general however we get a vanishing theorem in positive characteristic only for those points $\mathfrak{p} \in \operatorname{Spec} D$ for which the sheaf of relations $\mathcal{R}_{\mathfrak{p}}$ is strongly semistable.

Theorem 8.4. Suppose the situation and notation of Situation 7.1. Set $k=$ $\left\lceil\frac{d_{1}+\ldots+d_{n}}{n-1}\right\rceil$. Suppose that for the generic fiber the sheaf of relations is semistable. Then for all $p \gg 0$ such that the corresponding sheaf of relations is strongly semistable we have

$$
\left(f_{1}, \ldots, f_{n}\right)^{*}=\left(f_{1}, \ldots, f_{n}\right)+R_{\geq k} .
$$

Proof. The inclusion $\supseteq$ follows for every $\mathfrak{p} \in \operatorname{Spec} D$ such that the sheaf $\mathcal{R}_{\mathfrak{p}}(m)$ is strongly semistable on $Y_{\bar{\kappa}(\mathfrak{p})}$ from Theorem 6.4 .

For the inclusion $\subseteq$ we do not need the condition strongly semistable. From Theorem 7.3 we know that a single fixed element $f_{0}$ with $f_{0} \in\left(f_{1}, \ldots, f_{n}\right)^{*}$ also belongs to the right-hand side for $p \gg 0$, but here we state the identity of the two 
ideals for $p \gg 0$. Let $I=\left(f_{1}, \ldots, f_{n}\right)$ in $S$. We may assume by shrinking $D$ that $I=S \cap I S_{Q(D)}$. Let $m<k$ and consider $S_{m} / I_{m} \subseteq H^{1}\left(D\left(S_{+}\right), \operatorname{Rel}\left(f_{1}, \ldots, f_{n}\right)_{m}\right)$. We may assume that $S_{m} / I_{m}$ is a free $D$-module with a basis induced by $h_{j} \in S_{m}$, $1 \leq j \leq t$.

For every field $Q(D) \subseteq L$ the sheaf $\mathcal{F}_{L}(-m)=\mathcal{R}_{L}(m)^{\vee}$ is ample on Proj $S_{L}$ due to Theorem 2.3 hence for every $h=\sum \lambda_{j} h_{j} \neq 0$ the extension $\mathcal{F}_{L}^{\prime}(-m)(h)$ is also ample and the open subset $\mathbb{P}\left(\mathcal{F}_{L, h}^{\prime}\right)-\mathbb{P}\left(\mathcal{F}_{L}\right)$ is affine. This is then also true for the open subset $U_{L}=D\left(\left(S_{L}\right)_{+}\right) \subseteq$ Spec $S_{L}\left[T_{1}, \ldots, T_{n}\right] /\left(f_{1} T_{1}+\ldots+f_{n} T_{n}+h\right)$.

We introduce indeterminates $\Lambda_{j}, 1 \leq j \leq t$, for the coefficients of an element $h=\sum_{j} \lambda_{j} h_{j}$ and consider the universal forcing algebra

$$
C=S\left[T_{i}, \Lambda_{j}\right] /\left(f_{1} T_{1}+\ldots+f_{n} T_{n}+\Lambda_{1} h_{1}+\ldots+\Lambda_{t} h_{t}\right)
$$

over $S\left[\Lambda_{j}\right]$ and over $D\left[\Lambda_{j}\right]$. Let $U=D\left(S_{+}\right) \subseteq$ Spec $C$.

We claim that (after shrinking $D$ ) for every point $P \in \operatorname{Spec} D\left[\Lambda_{1}, \ldots, \Lambda_{t}\right], P \notin$ $V\left(\Lambda_{1}, \ldots, \Lambda_{t}\right)$, the fiber $U_{P}$ is affine. We show this by increasing inductively the open subset where this statement holds.

For the quotient field $L=Q(D)\left(\Lambda_{1}, \ldots, \Lambda_{t}\right)=Q\left(D\left[\Lambda_{1}, \ldots, \Lambda_{t}\right]\right)$ we know that $U_{L}$ is affine. Therefore $U \cap D(g)$ is also affine (by Remark 7.2), where $0 \neq g \in$ $D\left[\Lambda_{1}, \ldots, \Lambda_{t}\right]$, and we know then that the fiber $U_{P}$ is affine for every point $P \in$ $D(g) \in \operatorname{Spec} D\left[\Lambda_{1}, \ldots, \Lambda_{t}\right]$. So we know that the claim is true for a non-empty open subset.

For the induction step suppose that the claim is true for the open subset $W \subseteq$ Spec $D\left[\Lambda_{1}, \ldots, \Lambda_{t}\right]$. By shrinking $D$ we may assume that all irreducible components of the complement of $W$ dominate Spec $D$. Consider such an irreducible component $Z=V(\mathfrak{q})$ of maximal dimension and suppose that $Z \neq V\left(\Lambda_{1}, \ldots, \Lambda_{t}\right)$. The generic point $\zeta$ of $Z$ has characteristic zero and the $\Lambda_{j}$ are not all zero in $\kappa(\zeta)$, hence again $U_{\zeta}$ is affine and we find an open neighborhood $\zeta \in D(g) \cap Z \subseteq Z, g \in D\left[\Lambda_{1}, \ldots, \Lambda_{t}\right]$, such that for every point $P \in D(g) \cap Z$ the fiber $U_{P}$ is affine. Hence the claim is now true on a bigger open subset and the number of components of the complement of maximal dimension has dropped.

So we see that the claim holds eventually for $D\left(\Lambda_{1}, \ldots, \Lambda_{t}\right)$. Now the claim means in particular that for every prime ideal $\mathfrak{p} \in \operatorname{Spec} D$ and every linear combination $h=\sum_{j} \lambda_{j} h_{j} \neq 0, \lambda_{j} \in \kappa(\mathfrak{p})$, the corresponding open subset $U_{\mathfrak{p}, h}$ is affine, hence $h \notin I_{\kappa(\mathfrak{p})}^{*}$ for all $h \notin I_{\kappa(\mathfrak{p})}$.

This procedure can be done for every degree $0 \leq m<k$, hence we find a sufficiently small Spec $D$ such that the statement holds for all $\mathfrak{p} \in \operatorname{Spec} D$.

Remark 8.5. Theorem 8.1, Corollary 8.3 and Theorem 8.4 indicate that the number $\left\lceil\frac{d_{1}+\ldots+d_{n}}{n-1}\right\rceil$ is the generic bound for the degree of an element to belong to the tight closure; see also Theorem 9.1. It is reasonable to guess that for $R$ of higher dimension the number $\left\lceil\frac{\operatorname{dim} R-1}{n-1}\left(d_{1}+\ldots+d_{n}\right)\right\rceil$ should take over this part $(n \geq$ $\operatorname{dim} R)$.

The following result of Huneke and Smith - the vanishing theorem for parameters in dimension two - is an easy corollary of Theorems 8.1 and 8.4, see [17, Theorem 4.3].

Corollary 8.6. Let the notation be as in Situation 6.1 and suppose that $n=2$, so we are concerned with the tight closure of a parameter ideal. Suppose that the 
characteristic of $K$ is 0 or $p>\frac{2}{\delta}(g-1)$. Then

$$
\left(f_{1}, f_{2}\right)^{*}=\left(f_{1}, f_{2}\right)+R_{\geq d_{1}+d_{2}} .
$$

Proof. The statement for characteristic 0 and characteristic $p \gg 0$ follows at once from Theorems 8.1 and 8.4. since the sheaf of relations $\mathcal{R}$ is invertible, hence (strongly) semistable.

For the precise statement in positive characteristic we need the ampleness criterion of Hartshorne-Mumford for bundles of rank two; see Theorem 2.7. The inclusion $\supseteq$ is true in any characteristic by Theorem 6.4 since $\mathcal{R}(m)=\mathcal{O}_{Y}\left(m-d_{1}-d_{2}\right)$. So suppose that $f_{0} \in\left(f_{1}, f_{2}\right)^{*}$, but $f_{0} \notin\left(f_{1}, f_{2}\right)$ and $m=\operatorname{deg}\left(f_{0}\right)<d_{1}+d_{2}$. The corresponding forcing class $c \in H^{1}(Y, \mathcal{R}(m))$ gives the forcing sequence

$$
0 \longrightarrow \mathcal{O}_{Y} \longrightarrow \mathcal{F}^{\prime}(-m) \longrightarrow \mathcal{F}(-m) \longrightarrow 0 \text {. }
$$

Now $\operatorname{deg}\left(\mathcal{F}^{\prime}(-m)\right)=\left(d_{1}+d_{2}-m\right) \delta \geq \delta$, hence $\operatorname{deg}\left(\mathcal{F}^{\prime}(-m)\right)>\frac{2}{p}(g-1)$. This gives the first condition in Theorem 2.7. For the second condition, let $\mathcal{F}^{\prime}(-m) \rightarrow \mathcal{M} \rightarrow 0$ be an invertible quotient sheaf. If $\operatorname{deg}(\mathcal{M})<0$ or if $\operatorname{deg}(\mathcal{M})=0$ and $\mathcal{M} \neq \mathcal{O}_{Y}$, then the composed mapping $\mathcal{O}_{Y} \rightarrow \mathcal{M}$ is zero and $\mathcal{M}$ is a quotient of the ample invertible sheaf $\mathcal{F}(-m)$, which is not possible. If $\mathcal{M}=\mathcal{O}_{Y}$, then again the composed mapping $\mathcal{O}_{Y} \rightarrow \mathcal{O}_{Y}$ is not zero, hence it is an isomorphism and the sequence splits, which contradicts the assumption $c \neq 0$.

Remark 8.7. If $Y$ is a smooth plane curve given by a polynomial $F$ of degree $\delta$, then $g=(\delta-1)(\delta-2) / 2$ and the condition in Corollary 8.6 is that $p>\frac{2}{\delta}\left(\frac{(\delta-1)(\delta-2)}{2}-1\right)=$ $\delta-3$.

The condition in [17 Theorem 4.3] for Corollary 8.6 to hold in positive characteristic is that $p$ exceeds the degree of each of a set of homogeneous generators for the module of $k$-derivations of $R$.

\section{EXAmPLes FOR $n=3$}

In this last section we discuss the case where $n=3$. This means that we have three homogeneous elements $f_{1}, f_{2}, f_{3} \in R$ of degree $d_{1}, d_{2}, d_{3}$, where $R$ is our two-dimensional, normal standard-graded domain over an algebraically closed field. Then the sheaf of relations has rank two. If it is decomposable, then we are in the situation of Theorem [7.5 and the tight closure $\left(f_{1}, f_{2}, f_{3}\right)^{*}$ is easy to compute; see Example 9.7 below. So we shall concentrate on the indecomposable case. We will restrict to characteristic 0 ; for positive characteristic we may obtain with the help of Proposition 7.8 results which are slightly worse.

Theorem 9.1. Let $K$ denote an algebraically closed field of characteristic 0 and let $R$ denote a normal standard-graded $K$-domain of dimension two. Let $Y=\operatorname{Proj} R$ denote the corresponding smooth projective curve of genus $g$ and let $\delta$ denote the degree of $\mathcal{O}_{Y}(1)$. Let $f_{1}, f_{2}, f_{3}$ denote $R_{+}$-primary homogeneous elements of degree $d_{1}, d_{2}, d_{3}$ and suppose that the sheaf of relations $\mathcal{R}(m)$ is indecomposable on $Y$. Then

$$
\mu_{\max }\left(f_{1}, f_{2}, f_{3}\right) \leq \delta \frac{d_{1}+d_{2}+d_{3}}{2}+g-1
$$

and

$$
\mu_{\min }\left(f_{1}, f_{2}, f_{3}\right) \geq \delta \frac{d_{1}+d_{2}+d_{3}}{2}-g+1
$$


Proof. The sheaf $\mathcal{F}(-m)=\mathcal{R}^{\vee}(m)$ has rank two, hence $\mathbb{P}(\mathcal{F})$ is a ruled surface over $Y$. Since $\mathcal{F}(-m)$ is supposed to be indecomposable, we know by [13, Theorem V.2.12] that the $e$-invariant of $\mathbb{P}(\mathcal{F})$ is $e \leq 2 g-2$. If $\mathcal{F}(-m)$ is semistable, then the result is true, since then $\mu_{\max }(\mathcal{F}(0))=\mu(\mathcal{F}(0))=\delta\left(d_{1}+d_{2}+d_{3}\right) / 2$ and $g \geq 1$, since $\mathcal{F}(0)$ is indecomposable.

So suppose that $\mathcal{F}(0)$ is not semistable, set $\mathcal{F}=\mathcal{F}(0)$ and let $0 \rightarrow \mathcal{L} \rightarrow \mathcal{F} \rightarrow$ $\mathcal{Q} \rightarrow 0$ be a short exact sequence such that $\operatorname{deg}(\mathcal{L})=\mu(\mathcal{L})=\mu_{\max }(\mathcal{F})$. Then $\mathcal{F} \otimes \mathcal{L}^{-1}$ is normalized, since it has a section $\neq 0$ and $H^{0}\left(Y, \mathcal{F} \otimes \mathcal{L}^{-1} \otimes \mathcal{M}\right)=0$ for every invertible sheaf $\mathcal{M}$ of $\operatorname{deg}(\mathcal{M})<0$. For otherwise there would exist a non-trivial morphism $\mathcal{L} \otimes \mathcal{M}^{-1} \rightarrow \mathcal{F}$ which contradicts $\mu_{\max }(\mathcal{F})=\mu(\mathcal{L})$. Hence

$$
-e=\operatorname{deg}\left(\mathcal{F} \otimes \mathcal{L}^{-1}\right)=2 \mu\left(\mathcal{F} \otimes \mathcal{L}^{-1}\right)=2 \mu(\mathcal{F})+2 \mu\left(\mathcal{L}^{-1}\right)=2 \mu(\mathcal{F})-2 \mu(\mathcal{L})
$$

and therefore

$$
\mu_{\max }(\mathcal{F})=\mu(\mathcal{L})=\mu(\mathcal{F})+\frac{e}{2} \leq \delta \frac{d_{1}+d_{2}+d_{3}}{2}+\frac{2 g-2}{2} .
$$

The bound for $\mu_{\min }\left(f_{1}, f_{2}, f_{3}\right)$ follows from $\mu_{\min }(\mathcal{F})=2 \mu(\mathcal{F})-\mu_{\max }(\mathcal{F})$.

Corollary 9.2. Let $K$ denote an algebraically closed field of characteristic 0 and let $R$ denote a normal standard-graded $K$-domain of dimension two. Let $Y=$ Proj $R$ denote the corresponding smooth projective curve of genus $g$ and let $\delta$ denote the degree of $\mathcal{O}_{Y}(1)$. Let $f_{1}, f_{2}, f_{3}$ denote $R_{+}$-primary homogeneous elements of degree $d_{1}, d_{2}, d_{3}$ and suppose that the sheaf of relations $\mathcal{R}$ is indecomposable on $Y$. Then the following statements hold:

(i) For $m \geq \frac{d_{1}+d_{2}+d_{3}}{2}+\frac{g-1}{\delta}$ we have the inclusion $R_{m} \subseteq\left(f_{1}, f_{2}, f_{3}\right)^{*}$.

(ii) For $m<\frac{d_{1}+d_{2}+d_{3}}{2}-\frac{g-1}{\delta}$ we have $\left(f_{1}, f_{2}, f_{3}\right)^{*} \cap R_{m}=\left(f_{1}, f_{2}, f_{3}\right) \cap R_{m}$.

Proof. The first statement follows from Theorem 9.1 and from Theorem 6.4. The second statement follows from Theorem 9.1 and Theorem 7.3

Corollary 9.3. Let $K$ denote an algebraically closed field of characteristic 0 and let $F \in K[x, y, z]$ denote a homogeneous polynomial of degree $\delta$ such that $R=$ $K[x, y, z] /(F)$ is a normal domain. Let $f_{1}, f_{2}, f_{3} \in R$ denote $R_{+}$-primary homogeneous elements of degree $d_{1}, d_{2}, d_{3}$. Suppose that the sheaf of relations $\mathcal{R}$ is indecomposable on the curve $Y=\operatorname{Proj} R$. Then the following hold:

(i) $R_{m} \subseteq\left(f_{1}, f_{2}, f_{3}\right)^{*}$ for $m \geq \frac{d_{1}+d_{2}+d_{3}}{2}+\frac{\delta-3}{2}$.

(ii) For $m<\frac{d_{1}+d_{2}+d_{3}}{2}-\frac{\delta+3}{2}$ we have $\left(f_{1}, f_{2}, f_{3}\right)^{*} \cap R_{m}=\left(f_{1}, f_{2}, f_{3}\right) \cap R_{m}$.

Proof. This follows from Corollary 9.2 taking into account the fact that $g=(d-1)(d-2) / 2$.

We also have the following result in positive characteristic.

Corollary 9.4. Suppose the relative setting of Situation 7.1 and suppose that $n=3$. Suppose that $\mathcal{R}(m)$ is indecomposable on $Y_{\bar{\eta}}$, where $\eta$ is the generic point. Then for $p \gg 0$ we have

$$
\bar{\mu}_{\max }\left(f_{1}, f_{2}, f_{3}\right)<\left\lfloor\delta \frac{d_{1}+d_{2}+d_{3}}{2}\right\rfloor+g
$$

and

$$
\bar{\mu}_{\min }\left(f_{1}, f_{2}, f_{3}\right)>\left\lfloor\delta \frac{d_{1}+d_{2}+d_{3}}{2}\right\rfloor-g
$$


Proof. We have from Proposition 7.8 and Theorem 9.1 the estimates

$$
\begin{aligned}
\bar{\mu}_{\max }\left(f_{1}, f_{2}, f_{3}\right) & <\left\lfloor\mu_{\max }\left(\mathcal{F}_{\eta}(0)\right)\right\rfloor+1 \\
& \leq\left\lfloor\delta \frac{d_{1}+d_{2}+d_{3}}{2}+g-1\right\rfloor+1 \\
& =\left\lfloor\delta \frac{d_{1}+d_{2}+d_{3}}{2}\right\rfloor+g .
\end{aligned}
$$

Example 9.5. Let $F \in K[x, y, z]$ denote a homogeneous form of degree $\delta=5$ such that $R=K[x, y, z] /(F)$ is a normal domain. Suppose that $f_{1}, f_{2}, f_{3} \in R$ are primary homogeneous elements of degree 100 and such that its sheaf of relations is indecomposable. Then the bounds of Smith in [26] give $R_{\geq 200} \subseteq\left(f_{1}, f_{2}, f_{3}\right)^{*}$ on the one hand and, on the other hand, that an element of degree $m \leq 100$ belongs to $\left(f_{1}, f_{2}, f_{3}\right)^{*}$ if and only if it belongs to the ideal itself.

Our bounds in Corollary 9.3 give $R \geq 151 \subseteq\left(f_{1}, f_{2}, f_{3}\right)^{*}$ and that an element of degree $\leq 148$ belongs to $\left(f_{1}, f_{2}, f_{3}\right)^{*}$ if and only if it belongs to the ideal itself (for zero characteristic). So only the degrees 149 and 150 are not covered by Corollary 2.3. If furthermore the sheaf of relations is semistable, then Theorem 8.1 gives a complete numerical answer.

Remark 9.6. It seems not so easy to establish the semistability property for the relation bundle on a given curve of genus $\geq 2$. There exist however restriction theorems saying that this property holds on a generic curve of sufficiently high degree under the condition that the bundle is (defined and) semistable on the projective plane. We will come back to this kind of question in another paper.

Our last example gives a negative answer to a question of Craig Huneke asked at MSRI (September 2002). The example gives an ideal which is generated by $*-$ independent elements (meaning that none of them is contained in the tight closure of the others; see 29] for this notion), but it does not hold a vanishing theorem for it, that is, there does not exist a common inclusion and exclusion bound for tight closure.

Example 9.7. Consider the ideal $I=\left(x^{4}, x y, y^{2}\right)$ in the Fermat cubic $x^{3}+y^{3}+$ $z^{3}=0$. These ideal generators come from the regular polynomial ring $K[x, y] \subset$ $K[x, y, z] /\left(x^{3}+y^{3}+z^{3}\right)=R$. From this it follows that they are $*$-independent in $R$ and that the relation bundle must split, and in fact

$$
\mathcal{R}\left(x^{4}, x y, y^{2}\right)(5)=\mathcal{O}_{Y}(0) \oplus \mathcal{O}_{Y}(2),
$$

where $Y=\operatorname{Proj} R$ is the corresponding elliptic curve. Let $h$ denote a homogeneous element of degree $m$, given rise to a forcing class

$$
c \in H^{1}(Y, \mathcal{R}(m))=H^{1}\left(Y, \mathcal{O}_{Y}(m-5)\right) \oplus H^{1}\left(Y, \mathcal{O}_{Y}(m-3)\right) .
$$

From the numerical criterion in Theorem 7.5 it is easy to deduce the following:

For $m \geq 5$ we have $R_{m} \subset I^{*}$.

For $m=4$ an element $h$ belongs to $I^{*}$ only if it belongs to $I$.

For $m=3$ all possibilities occur. We find $y z^{2} \in\left(x^{4}, x y, y^{2}\right)^{*}$, but not in the ideal itself, and $x z^{2} \notin I^{*}$. 


\section{REFERENCES}

[1] A. Alzati, M. Bertolini, G. M. Besana, Numerical criteria for very ampleness of divisors on projective bundles over an elliptic curve, Can. J. Math. 48 (6) (1996), 1121-1137. MR 97k:14032

[2] C. M. Barton, Tensor products of ample vector bundles in characteristic $p$, Am. J. Math. 93 (1971), 429-438. MR 44:6713

[3] H. Brenner, Tight closure and projective bundles, J. Algebra 265 (2003), 45-78.

[4] H. Brenner, Tight closure and plus closure for cones over elliptic curves, submitted.

[5] F. Campana and H. Flenner, A characterization of ample vector bundles on a curve, Math. Ann. 287 (1990), 571-575. MR 91f:14027

[6] D. Gieseker, p-ample bundles and their chern classes, Nagoya Math. J. 43 (1971), 91-116. MR 45:5139

[7] A. Grothendieck and J. Dieudonné, Eléments de géométrie algébrique II, Inst. Hautes Études Sci. Publ. Math. 8 (1961). MR 36:177b

[8] A. Grothendieck and J. Dieudonné, Eléments de géométrie algébrique III, Inst. Hautes Études Sci. Publ. Math. 11 (1961). MR 36:177c

[9] G. Harder, M. S. Narasimhan, On the cohomology groups of moduli spaces of vector bundles on curves, Math. Ann. 212 (1975), 215-248. MR 51:509

[10] R. Hartshorne, Ample vector bundles, Publ. Math. I.H.E.S. 29 (1966), 63-94. MR 33:1313

[11] R. Hartshorne, Ample vector bundles on curves, Nagoya Math. J. 43 (1971), 73-89. MR 45:1929

[12] R. Hartshorne, Ample Subvarieties of Algebraic Varieties, Springer-Verlag, Berlin-Heidelberg-New York, 1970. MR 44:211

[13] R. Hartshorne, Algebraic Geometry, Springer-Verlag, New York, 1977. MR 57:3116

[14] M. Hochster, Solid closure, Contemp. Math. 159 (1994), 103-172. MR 95a:13011

[15] C. Huneke, Tight Closure and Its Applications, AMS, 1996. MR 96m:13001

[16] C. Huneke, Tight Closure, Parameter Ideals, and Geometry, in Six Lectures on Commutative Algebra, Birkhäuser, Basel, 1998. MR 99j:13001

[17] C. Huneke, K. Smith, Tight closure and the Kodaira vanishing theorem, J. Reine Angew. Math. 484 (1997), 127-152. MR 98e:13007

[18] D. Huybrechts, M. Lehn, The Geometry of Moduli Spaces of Sheaves, Viehweg, Braunschweig, 1997. MR 98g:14012

[19] P. Ionescu and M. Toma, On very ample vector bundles on curves, Int. Jour. of Math. 8, No. 5 (1997), 633-643. MR 98h:14036

[20] H. Lange, Zur Klassifikation von Regelmannigfaltigkeiten, Math. Ann. 262 (1983), 447459. MR 85b:14019

[21] R. Lazarsfeld, Positivity in Algebraic Geometry (Preliminary Draft), 2001.

[22] Y. Miyaoka, The Chern class and Kodaira dimension of a minimal variety, in Algebraic Geometry, Sendai 1985, Adv. Stud. Pure Math. 10, 1987, 449-476. MR 89k:14022

[23] S. Mukai, F. Sakai, Maximal subbundles of vector bundles on a curve, Manuscripta math. 52 (1985), 251-256. MR 86k:14013

[24] C. Okonek, M. Schneider, and H. Spindler, Vector Bundles on Complex Projective Spaces, Birkhäuser, Boston, Basel, Stuttgart, 1980. MR 81b:14001

[25] C. S. Seshadri, Fibrés vectorielle sur les courbes algébrique, Asterisque 96, 1982. MR 85b:14023

[26] K. E. Smith, Tight closure in graded rings, J. Math. Kyoto Univ 37, No. 1 (1997), 35-53. MR 98e: 13009

[27] X. Sun, Remarks on semistability of $G$-bundles in positive characteristic, Comp. Math. 194 (1999), 41-52. MR 2001e:14032

[28] H. Tango, On the behaviour of extensions of vector bundles under the Frobenius map, Nagoya Math. J. 48 (1972), 73-89. MR 47:3401

[29] A. Vraciu, *-independence and special tight closure, J. Algebra 249, 2 (2002), 544-565. MR 2003d:13003

Mathematische Fakultät, Ruhr-Universität Bochum, 44780 Bochum, Germany

E-mail address: Holger.Brenner@ruhr-uni-bochum.de 